\title{
Bounds on the Electromagnetic Dipole Moments through the Single Top Production at the CLIC
}

\author{
M. Köksal, ${ }^{1}$ A. A. Billur, ${ }^{2}$ and A. Gutiérrez-Rodríguez ${ }^{3}$ \\ ${ }^{1}$ Department of Optical Engineering, Cumhuriyet University, 58140 Sivas, Turkey \\ ${ }^{2}$ Department of Physics, Cumhuriyet University, 58140 Sivas, Turkey \\ ${ }^{3}$ Facultad de Física, Universidad Autónoma de Zacatecas, Apartado Postal C-580, 98060 Zacatecas, ZAC, Mexico
}

Correspondence should be addressed to A. Gutiérrez-Rodríguez; alexgu@fisica.uaz.edu.mx

Received 11 September 2016; Revised 6 November 2016; Accepted 15 November 2016; Published 6 February 2017

Academic Editor: Lorenzo Bianchini

Copyright (C) 2017 M. Köksal et al. This is an open access article distributed under the Creative Commons Attribution License, which permits unrestricted use, distribution, and reproduction in any medium, provided the original work is properly cited. The publication of this article was funded by SCOAP ${ }^{3}$.

\begin{abstract}
We obtain bounds on the anomalous magnetic and electric dipole moments of the $t$-quark from a future high-energy and highluminosity linear electron positron collider, as the CLIC, with polarized and unpolarized electron beams which are powerful tools for determining new physics. We consider the processes $\gamma e^{-} \rightarrow \bar{t} b v_{e}(\gamma$ is the Compton backscattering photon) and $e^{+} e^{-} \rightarrow e^{-} \gamma^{*} e^{+} \rightarrow \bar{t} b v_{e} e^{+}\left(\gamma^{*}\right.$ is the Weizsacker-Williams photon) as they are one of the most important sources of single top quark production. For systematic uncertainties of $\delta_{\text {sys }}=0 \%(5 \%), b$-tagging efficiency $=0.8$, center-of-mass energy of $\sqrt{s}=3 \mathrm{TeV}$, and integrated luminosity of $\mathscr{L}=2 a b^{-1}$ the future $e^{+} e^{-}$collider may put bounds on the electromagnetic dipole moments $\widehat{a}_{V}$ and $\widehat{a}_{A}$ of the top quark of the order of $\mathcal{O}\left(10^{-2}-10^{-1}\right)$ at the $2 \sigma(3 \sigma)$ level, which are competitive with those recently reported in previous studies at hadron colliders and the ILC.
\end{abstract}

\section{Introduction}

The top quark is by far the heaviest particle of the Standard Model (SM) [1-3], with a mass of $m_{t}=173.5 \pm 0.6$ (stat.) \pm 0.8 (syst.) [4]. Up to now, the top quark has only been studied at the Tevatron and Large Hadron Collider (LHC). Its large mass implies that the top quark is the SM particle most strongly coupled to the mechanism of electroweak symmetry breaking. This is the principal reason it is considered to be one of the most likely places where new physics might be discovered. This means the top quark is a window to any new physics at the $\mathrm{TeV}$ energy scale. While much information about the top quark is already available that shows consistency with SM expectations, its properties and interactions are among the most important measurements for present and future high-energy colliders [5-16].

The construction of a high-energy $e^{+} e^{-}$International Linear Collider (ILC) has been proposed to complement direct searches carried out at the LHC. Precision measurements of top quark properties, in particular of its couplings, are especially interesting because the top quark is the heaviest known elementary particle and thus expected to be more sensitive to new physics at higher scales.

The top quark has been studied in some detail at the Tevatron and LHC. Many of its properties are still poorly constrained such as mass, spin, color and electric charges, the electric and magnetic dipole moments, and the chromomagnetic and chromoelectric dipole moments. Therefore, significant new insights on top quark properties will be one of the tasks of the LHC, the ILC $[7-10,15,16]$, and the Compact Linear Collider (CLIC) [12, 17].

The dipole moments of the top quark are some of the most sensitive observables, and although these intrinsic properties have been studied extensively both theoretically and experimentally, it is necessary to have more precise measurements. The dipole moments of the top quark have been investigated by several authors and in a variety of theoretical models [18-25]. Further, a number of studies show that, in the processes $e^{+} e^{-} \rightarrow t \bar{t}$ and $\gamma \gamma \rightarrow t \bar{t}$, the dipole moments of the top quark can be measured with great sensitivity [2629]. However, there are a significant number of top quarks that are produced in single form via the weak interaction. 
There are several single top quark production processes of interest in $e^{+} e^{-}, e^{-} e^{-}, \gamma e^{-}$and $\gamma \gamma$ collisions, characterized by the virtuality of the $W$ boson [30-39].

Although studying single top quark production may not be considered of great importance, there are several reasons why its study is necessary in future linear $e^{+} e^{-}$colliders: (1) It is a very good alternative to study the dipole moments $\widehat{a}_{V}$ and $\widehat{a}_{A}$ of the top quark, as well as the anomalous coupling $t b W$. (2) Single top production at CLIC in association with a $W$ boson and bottom quark through $W W^{*}$ production leads to the same final state as $t$ quark pair production. (3) The cross section for single top quark production processes is significant since production is abundant in $e^{+} e^{-}$colliders that operate at high energies. In addition, the single top quark production is directly proportional to the square of the $t b W$ coupling, and therefore it is potentially very sensitive to the $t b W$ structure [40]. (4) Single top quarks are produced with nearly $100 \%$ polarization due to the weak interaction [41, 42]. (5) New physics can influence single top production by inducing weak interactions beyond the SM weak interactions $[42,43]$, through loop effects [44-46], or by providing new sources of single top quark production [47-49]. For these reasons, it is important to study the properties of the top quark, in particular their dipole moments through the single top quark production processes.

In the SM, the prediction for the magnetic dipole moment (MDM) of the top quark is $a_{t}^{\mathrm{SM}}=0.02$ [50], which can be tested in current and future colliders, such as LHC and CLIC. In contrast, its electric dipole moment (EDM) is strongly suppressed and less than $10^{-30} e \mathrm{~cm}[18,51,52]$, which is too small to be observed. It is, however, highly attractive for probing new physics.

The sensitivity to the EDM has been studied in models with vector-like multiplets which predicted the top quark EDM close to $1.75 \times 10^{-3}$ [53].

There are studies performed via the $t \bar{t} \gamma$ production for the LHC at $\sqrt{s}=14 \mathrm{TeV}$ and $\mathscr{L}=300 \mathrm{fb}^{-1}$ and $3000 \mathrm{fb}^{-1}$, with limits of \pm 0.2 and \pm 0.1 , respectively [54]. Other limits are reported in the literature: $-2.0 \leq \widehat{a}_{V} \leq 0.3$ and $-0.5 \leq$ $\widehat{a}_{A} \leq 1.5$ which are obtained from the branching ratio and the $\mathrm{CP}$ asymmetry from radiative $b \rightarrow s \gamma$ transitions [55], while the bounds of $\left|\widehat{a}_{V}\right|<0.05(0.09)$ and $\left|\widehat{a}_{A}\right|<0.20(0.28)$ come from measurements of $\gamma p \rightarrow t \bar{t}$ cross section with 10\% (18\%) uncertainty, respectively [56]. More recent limits on the top quark magnetic and electric dipole moments through the process $p p \rightarrow p \gamma^{*} \gamma^{*} p \rightarrow p t \bar{t} p$ at the LHC with $\sqrt{s}=14 \mathrm{TeV}$, $\mathscr{L}=3000 \mathrm{fb}^{-1}$, and $68 \%$ C.L. are $-0.6389 \leq \widehat{a}_{V} \leq 0.0233$ and $\left|\widehat{a}_{A}\right| \leq 0.1158$ [57]. Sensitivity limits for the anomalous couplings of the top quark through the production process of top quark pairs $e^{+} e^{-} \rightarrow t \bar{t}$ for the ILC at $\sqrt{s}=500 \mathrm{GeV}$, $\mathscr{L}=200 \mathrm{fb}^{-1}, \mathscr{L}=300 \mathrm{fb}^{-1}$, and $\mathscr{L}=500 \mathrm{fb}^{-1}$ are predicted to be of the order of $\mathcal{O}\left(10^{-3}\right)$. Thus, the measurements at an electron positron collider lead to a significant improvement in comparison with LHC. Detailed discussions on the dipole moments of the top quark in top quark pairs production at the ILC are reported in the literature [7-11, 14-16, 26-29, 58$60]$. It is worth mentioning that there are no limits reported in the literature on the dipole moments $\widehat{a}_{V}$ and $\widehat{a}_{A}$ via single top quark production processes.
CP violation was first observed in a small fraction of $K$ mesons decaying to two pions in the SM. This phenomenology in the SM can be easily introduced by the CabibboKobayashi-Maskawa mechanism in the quark sector. For this reason, the presence of new physics beyond the SM can be investigated by examining the electromagnetic properties of the top quark that are defined with CP-symmetric and $\mathrm{CP}$-asymmetric anomalous form factors. Its dipole moments such as the MDM come from one-loop level perturbations and the corresponding EDM, which is described as a source of $\mathrm{CP}$ violation.

Following [54, 57, 61-63], the definition of the general effective coupling $t \bar{t} \gamma$, including the SM coupling and contributions from dimension-six effective operators, can be parameterized by the following effective Lagrangian:

$$
\mathscr{L}_{\gamma t \bar{t}}=-g_{e} Q_{t} \bar{t} \Gamma_{\gamma t \bar{t}}^{\mu} t A_{\mu},
$$

where $g_{e}$ is the electromagnetic coupling constant and $Q_{t}$ is the top quark electric charge and $\Gamma_{\gamma t \bar{t}}^{\mu}$ the Lorentz-invariant vertex function which describes the interaction of a $\gamma$ photon with two top quarks and can be parameterized by

$$
\Gamma_{\gamma t \bar{t}}^{\mu}=\gamma^{\mu}+\frac{i}{2 m_{t}}\left(\widehat{a}_{V}+i \widehat{a}_{A} \gamma_{5}\right) \sigma^{\mu \nu} q_{v},
$$

where $m_{t}$ is the mass of the top quark, $q$ is the momentum transfer to the photon, and the couplings $\widehat{a}_{V}$ and $\widehat{a}_{A}$ are real and related to the anomalous magnetic moment and the electric dipole moment of the top quark, respectively.

The majority of physics research in linear colliders is done assuming positron and electron beams are unpolarized. However, another significant advantage of the linear colliders is to obtain suitability of a highly polarized electron beam that can be polarized up to $\pm 80 \%$. A polarized electron beam provides a method to investigate the SM and to diagnose new physics beyond the SM. Observation of even the tiniest signal which conflicts with the SM expectations would be persuasive evidence for new physics. Proper selection of the electron beam polarization may therefore be used to enhance the new physics signal and also to considerably suppress backgrounds.

In this work we study the sensibility of the anomalous magnetic and electric dipole moments of the top quark through the processes $\gamma e^{-} \rightarrow \bar{t} b v_{e}$ ( $\gamma$ is the Compton backscattering photon) and $e^{+} e^{-} \rightarrow e^{-} \gamma^{*} e^{+} \rightarrow \bar{t} b v_{e} e^{+}\left(\gamma^{*}\right.$ is the Weizsacker-Williams photon) which are among the most important sources of single top quark production [30,33]. We use center-of-mass energies of the CLIC [17]. These values are for a center-of-mass energy of $1.4 \mathrm{TeV}$ with integrated luminosity of $1500 \mathrm{fb}^{-1}$ and $3 \mathrm{TeV}$ with $\mathscr{L}=2000 \mathrm{fb}^{-1}$ and polarized and unpolarized electron beams $P_{e^{-}}=-80 \%$ and $P_{e^{+}}=0 \%$ [64]. Not only can the future $e^{+} e^{-}$linear collider be designed to operate in $e^{+} e^{-}$collision mode, but also it can be operated as $e \gamma$ and $\gamma \gamma$ collider. This is achieved by using Compton backscattered photons in the scattering of intense laser photons on the initial $e^{+} e^{-}$beams. Another well-known application of linear colliders is to study new physics beyond the SM through $e \gamma^{*}$ and $\gamma^{*} \gamma^{*}$ collisions. A quasireal $\gamma^{*}$ photon emitted from one of the incoming 
$e^{-}$or $e^{+}$beams interacts with the other lepton shortly after generating the subprocess $\gamma^{*} e^{-} \rightarrow \bar{t} b v_{e}$. Hence, first we calculate the main reaction $e^{+} e^{-} \rightarrow e^{-} \gamma^{*} e^{+} \rightarrow \bar{t} b v_{e} e^{+}$by integrating the cross section for the subprocess $\gamma^{*} e^{-} \rightarrow \bar{t} b v_{e}$. In this case, the quasireal photons in $\gamma^{*} e^{-}$collisions can be examined by Equivalent Photon Approximation (EPA) [6567] using the Weizsacker-Williams approximation (WWA). In EPA, photons emitted from incoming leptons which have very low virtuality are scattered at very small angles from the beam pipe. These emitted quasireal photons have a low $Q^{2}$ virtuality and are therefore almost real. We only use the photon virtuality of $Q_{\max }^{2}=2 \mathrm{GeV}^{2}$. Also, we can add parts related to the large values of $Q_{\max }^{2}$ which do not significantly contribute to obtaining sensitivity limits on the anomalous couplings [68-71]. These processes have been observed phenomenologically and experimentally at the LEP, Tevatron, and LHC [72-93].

Taking all of the aforementioned into account, we study the potential of the processes $\gamma e^{-} \rightarrow \bar{t} b v_{e}$ and $e^{+} e^{-} \rightarrow$ $e^{+} \gamma^{*} e^{-} \rightarrow \bar{t} b v_{e} e^{+}$via Compton backscattering and WWA, respectively, and derive bounds on the dipole moments $\widehat{a}_{V}$ and $\widehat{a}_{A}$ at $2 \sigma$ and $3 \sigma$ level $(90 \%$ and $95 \%$ C.L.) and at a future high-energy and high-luminosity linear electron positron collider, as the CLIC, to study the sensibility on the anomalous magnetic and electric dipole moments of the top quark. The corresponding schematic and Feynman diagrams for the main reactions as well as for the subprocesses which give the most significant contribution to the total cross section are shown in Figures 1 and 2.

This paper is organized as follows. In Section 2, we study the dipole moments of the top quark through the process $\gamma e^{-} \rightarrow \bar{t} b v_{e}$ and in Section 3, through the process $e^{+} e^{-} \rightarrow$ $e^{+} \gamma^{*} e^{-} \rightarrow \bar{t} b v_{e} e^{+}$. Finally, we summarize our conclusions in Section 4 .

\section{Compton Backscattering: Cross Section of $\gamma e^{-} \rightarrow \bar{t} b v_{e}$}

In this section we present numerical results of the cross section for the process $\gamma e^{-} \rightarrow \bar{t} b v_{e}$, using the CalcHEP [94] packages for calculations of the matrix elements and cross sections. These packages provide automatic computation of the cross sections and distributions in the SM as well as their extensions at tree level. We consider the high-energy stage of possible future linear $\gamma e^{-}$collisions with $\sqrt{s}=1.4$ and $3 \mathrm{TeV}$ and design luminosity of 50, 300, 500, 1000, 1500, and $2000 \mathrm{fb}^{-1}$ according to the new data reported by the CLIC [17]. In addition, in all numerical analysis we consider the $b$ tagging efficiency of 0.8 and systematic uncertainty of $\delta_{\text {sys }}=$ $0 \%, 5 \%$ and the acceptance cuts will be imposed as $\left|\eta^{b}\right|<2.5$ for pseudorapidity and $p_{T}^{b}>20 \mathrm{GeV}$ and $p_{T}^{v_{e}}>10 \mathrm{GeV}$ for transverse momentums of the final state particles. We also consider the hadronic decay channels of the top quark BR = 0.676 (hadronic branching ratio). There are systematic uncertainties for hadron colliders for single top quark production [95]. For example, these uncertainties arise from luminosity, jet identification, backgrounds, $b$-tagging efficiency. On the other hand, linear colliders have less uncertainties with

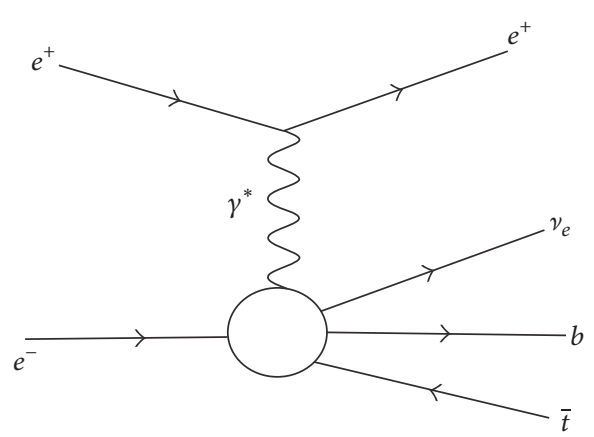

Figure 1: Schematic diagram for the process of single top quark production $e^{+} e^{-} \rightarrow e^{+} \gamma^{*} e^{-} \rightarrow \bar{t} b v_{e} e^{+}$.

respect to hadron colliders for determination of the cross section of single top quark production [96]. Therefore, for events estimation in $\chi^{2}$ analysis, we have taken into account $b$-tagging efficiency and consider systematic uncertainties of $0 \%$ and $5 \%$. The values close to this systematic uncertainty value have been taken into account in previous studies; for example, in [97], a 3\% systematic error in the total cross section has been assumed for the $e^{-} e^{+} \rightarrow t \bar{t}$ process at the ILC. It can be seen that the systematic error in the cross section determination has been lowered from $3 \%$ to $1 \%$ [98]. However, since there is no study related to the systematic error on the single top quark production at the CLIC, we use systematic errors of $0 \%$ and $5 \%$ for the processes studied in this paper.

In our study we examined the projected $2 \sigma$ and $3 \sigma$ sensitivities on the dipole moments $\widehat{a}_{V}$ and $\widehat{a}_{A}$ of the top quark for the processes $\gamma e^{-} \rightarrow \bar{t} b v_{e}(\gamma$ is the Compton backscattering photon) and $e^{+} e^{-} \rightarrow e^{-} \gamma^{*} e^{+} \rightarrow \bar{t} b v_{e} e^{+}\left(\gamma^{*}\right.$ is the WeizsackerWilliams photon) at the CLIC-1.4 TeV and CLIC- $3 \mathrm{TeV}$, respectively. We use the chi-squared distribution test defined as

$$
\chi^{2}=\left(\frac{\sigma_{\mathrm{SM}}-\sigma_{\mathrm{NP}}\left(\widehat{a}_{V}, \widehat{a}_{A}\right)}{\sigma_{\mathrm{SM}} \delta}\right)^{2},
$$

where $\sigma_{\mathrm{NP}}\left(\widehat{a}_{V}, \widehat{a}_{A}\right)$ is the total cross section including contributions from the SM and new physics, $\delta=\sqrt{\left(\delta_{\text {st }}\right)^{2}+\left(\delta_{\text {sys }}\right)^{2}}$, $\delta_{\text {st }}=1 / \sqrt{N_{\mathrm{SM}}}$ is the statistical error, $\delta_{\text {sys }}$ is the systematic error, and $N_{\mathrm{SM}}$ is the number of signal expected events $N_{\mathrm{SM}}=$ $\mathscr{L}_{\text {int }} \times \mathrm{BR} \times \sigma_{S \mathrm{M}} \times \epsilon_{b}$, where $\epsilon_{b}=0.8$ is the $b$-tagging efficiency and $\mathscr{L}_{\text {int }}$ is the integrated CLIC luminosity.

2.1. Top Quark Dipole Moments through the Process $\gamma e^{-} \rightarrow$ $\bar{t} b v_{e}$ with Polarized and Unpolarized Beams. With polarized beams of electrons and positrons, the cross section of a process can be expressed as [64]

$$
\begin{aligned}
& \sigma\left(P_{e^{-}}, P_{e^{+}}\right)=\frac{1}{4}\left[\left(1+P_{e^{-}}\right)\left(1+P_{e^{+}}\right) \sigma_{++}\right. \\
& \quad+\left(1-P_{e^{-}}\right)\left(1-P_{e^{+}}\right) \sigma_{--}+\left(1+P_{e^{-}}\right)\left(1-P_{e^{+}}\right) \sigma_{+-} \\
& \left.\quad+\left(1-P_{e^{-}}\right)\left(1+P_{e^{+}}\right) \sigma_{-+}\right],
\end{aligned}
$$




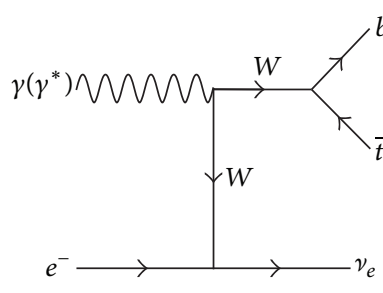

(1)

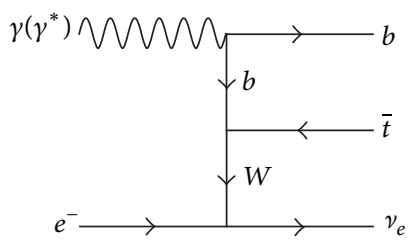

(3)

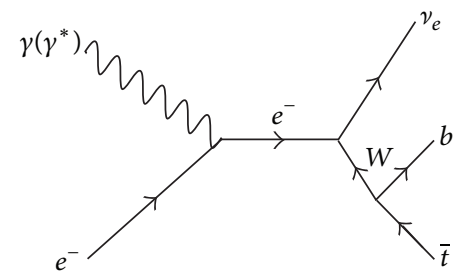

(2)

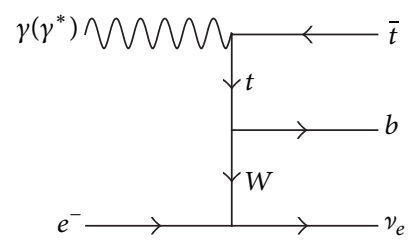

(4)

FIGURE 2: The Feynman diagrams contributing to the process $\gamma e^{-} \rightarrow \bar{t} b v_{e}$ and the subprocess $\gamma^{*} e^{-} \rightarrow \bar{t} b v_{e}$.

where $P_{e^{-}}\left(P_{e^{+}}\right)$is the polarization degree of the electron (positron) beam, while $\sigma_{-+}$stands for the cross section for completely left-handed polarized $e^{-}$beam $P_{e^{-}}=-1$ and completely right-handed polarized $e^{+}$beam $P_{e^{+}}=1$, and other cross sections $\sigma_{--}, \sigma_{++}$and $\sigma_{+-}$are defined analogously.

The corresponding Feynman diagrams for the process $\gamma e^{-} \rightarrow \bar{t} b v_{e}$ that give the most important contribution to the total cross sections are shown in Figure 2. In this figure the Feynman diagrams (1)-(3) correspond to the contribution of the SM, while diagram (4) corresponds to the anomalous contribution; that is, for the $\gamma e^{-}$collisions there is SM background at the tree level so the total cross section is proportional to $\sigma_{\text {Tot }}=\sigma_{\mathrm{SM}}+\sigma_{\text {Int }}\left(\widehat{a}_{V}, \widehat{a}_{A}\right)+$ $\sigma_{\text {Anom }}\left(\widehat{a}_{V}^{2}, \widehat{a}_{A}^{2}, \widehat{a}_{V} \widehat{a}_{A}\right)$, respectively.

To illustrate our results, we show the dependence of the cross section on the anomalous couplings $\widehat{a}_{V}$ and $\widehat{a}_{A}$ for $\gamma e^{-} \rightarrow \bar{t} b v_{e}$ in Figure 3 for $P_{e^{-}}=-80 \%, P_{e^{+}}=0 \%$, as well as on unpolarized beams and two different center-ofmass energies $\sqrt{s}=1.4,3 \mathrm{TeV}$ [17], whereas the $\widehat{a}_{V}\left(\widehat{a}_{A}\right)$ anomalous coupling is kept fixed at zero. We observed that the cross section is sensitive to the value of the centerof-mass energies. The sensitivity to $\bar{t} b v_{e}$ increases with the collider energy reaching a maximum at the end of the range considered, $\widehat{a}_{V, A}= \pm 1$, and the cross section for $\sqrt{s}=$ $3 \mathrm{TeV}$ increases relative to $\sqrt{s}=1.4 \mathrm{TeV}$ up to $24.5 \%$ with polarized beams and up to $26.6 \%$ with unpolarized beams. By contrast, in the vicinity of $\widehat{a}_{V, A}=0$ the total cross section is smaller. We notice that, as shown in Figure 3, the $\gamma e^{-} \rightarrow \bar{t} b v_{e}$ production process at an CLIC-based $\gamma e^{-}$collider reaches a value of $\sigma=0.55 \mathrm{pb}(0.3 \mathrm{pb})$ for $\sqrt{s}=3 \mathrm{TeV}$ for polarized and unpolarized beams. Although the cross section for unpolarized beams is approximately half of that of polarized beams, in both cases the $t \bar{t} \gamma$ coupling could be probed with remarkable sensitivity (see Tables 1 and 2).

In Figure 4 we used two center-of-mass energies $\sqrt{s}=$ $1.4,3 \mathrm{TeV}$ expected for the CLIC accelerator in order to get contour limits in the plane $\widehat{a}_{V}-\widehat{a}_{A}$ for $\gamma e^{-} \rightarrow \bar{t} b v_{e}$ and the expected luminosities of $\mathscr{L}=50,500,1500,2000 \mathrm{fb}^{-1}$ with polarized and unpolarized beams of electrons and positrons.
As an indicator of the order of magnitude, using $b$-tagging efficiency of 0.8 and considering the systematic errors of $\delta_{\text {sys }}=0 \%, 5 \%$, in Tables 1 and 2 we present the bounds obtained on the $\widehat{a}_{V}$ magnetic moment and $\widehat{a}_{A}$ electric dipole moments of the $t$-quark with the polarization $P_{e^{-}}=-80 \%$ for the electron beams and $P_{e^{+}}=0 \%$ for the positron, as well as with unpolarized beams, with $\sqrt{s}=1.4,3 \mathrm{TeV}$, $\mathscr{L}=50,300,500,1000,1500,2000 \mathrm{fb}^{-1}$ at $2 \sigma$ and $3 \sigma$ C.L., respectively. As expected, the results presented in Tables 1 and 2 clearly show that as the energy and luminosity of the collider increase, the bounds on the dipole moments of the top quark are stronger. We observed that these results are competitive with those recently reported in previous studies [54-57]. From results presented in Table 1, it is obvious that the effect of polarized beams is more significant than the effect of unpolarized beams (see Table 2).

To complement our results, in Table 3 we show the single top production total cross section for the process $\gamma e^{-} \rightarrow \bar{t} b v_{e}$ as a function of the dipole moments $\widehat{a}_{V}$ and $\widehat{a}_{A}$ at the two CLIC energies of 1.4 and $3 \mathrm{TeV}$. For polarized beams $\left(P_{e^{-}, e^{+}}=\right.$ $-80 \%, 0 \%)$, the total cross section most significant for the process considered is $\sigma_{\mathrm{Tot}}\left(\gamma e^{-} \rightarrow \bar{t} b v_{e}\right)=5.4330 \times 10^{-1} \mathrm{pb}$ for $\widehat{a}_{V}=-1, \widehat{a}_{A}=0$, and $\sqrt{s}=3 \mathrm{TeV}$, while for $\widehat{a}_{A}=1, \widehat{a}_{V}=0$, and $\sqrt{s}=3 \mathrm{TeV}$ the total cross section is $\sigma_{\mathrm{Tot}}\left(\gamma e^{-} \rightarrow \bar{t} b v_{e}\right)=$ $5.3923 \times 10^{-1} \mathrm{pb}$. On the other hand, for unpolarized beams $\left(P_{e^{-}, e^{+}}=0 \%, 0 \%\right)$, the total cross sections are $\sigma_{\text {Tot }}\left(\gamma e^{-} \rightarrow\right.$ $\left.\bar{t} b v_{e}\right)=3.0186 \times 10^{-1} \mathrm{pb}$ for $\widehat{a}_{V}=-1, \widehat{a}_{A}=0$ and $\sigma_{\text {Tot }}\left(\gamma e^{-} \rightarrow\right.$ $\left.\bar{t} b v_{e}\right)=2.9953 \times 10^{-1} \mathrm{pb}$ for $\widehat{a}_{V}=0, \widehat{a}_{A}=-1$ with $\sqrt{s}=3 \mathrm{TeV}$, respectively. Therefore, the total cross section for the case of polarized beams shows improvement by a factor of 1.8 with respect to the unpolarized case.

\section{Weizsacker-Williams Approximation \\ (WWA): Cross Section of $e^{+} e^{-} \rightarrow e^{+} \gamma^{*} e^{-} \rightarrow$ $\bar{t} b v_{e} e^{+}$}

We use the WWA and consider the process $e^{+} e^{-} \rightarrow e^{+} \gamma^{*} e^{-} \rightarrow$ $\bar{t} b v_{e} e^{+}$which is potentially useful for studying the dipole 

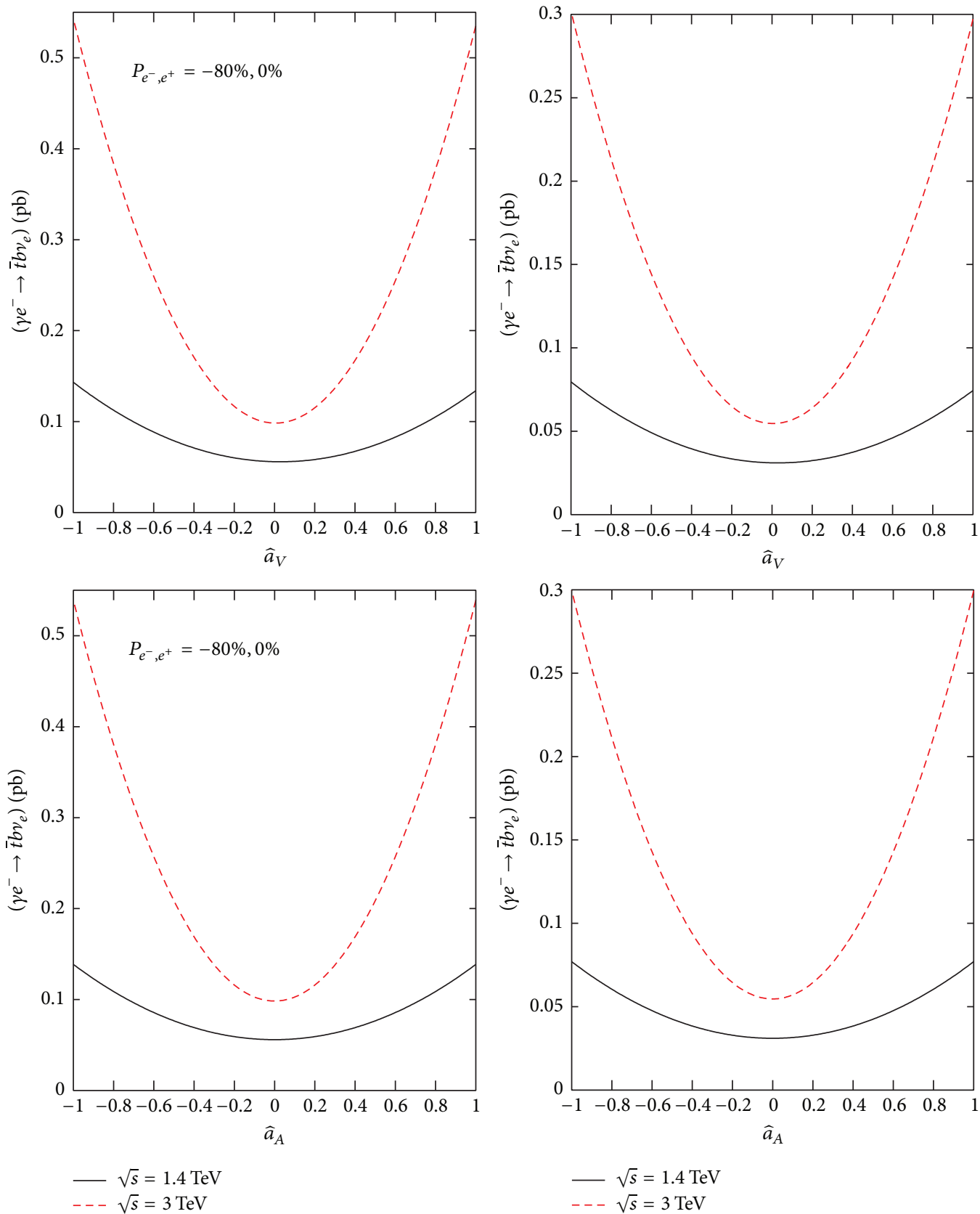

FIGURE 3: The integrated total cross section of the process $\gamma e^{-} \rightarrow \bar{t} b v_{e}\left(\gamma\right.$ is the Compton backscattering photon) as a function of $\hat{a}_{V}$ and $\widehat{a}_{A}$ with $\sqrt{s}=1.4,3 \mathrm{TeV}$ and polarized and unpolarized beams.

moments of the top quark with polarized and unpolarized $e^{-}$beams and for the center-of-mass energies of the CLIC [17].

3.1. Top Quark Dipole Moments through the Process $e^{+} e^{-} \rightarrow$ $e^{+} \gamma^{*} e^{-} \rightarrow \bar{t} b v_{e} e^{+}$with Polarized and Unpolarized Beams. The Feynman diagrams for the subprocess $\gamma^{*} e^{-} \rightarrow \bar{t} b v_{e}$ are shown in Figure 2. The total cross section of the subprocess depends on the contribution of the SM (diagrams (1)-(3)) plus the contribution of the anomalous couplings (diagram (4)).

For the study of the process $e^{+} e^{-} \rightarrow e^{+} \gamma^{*} e^{-} \rightarrow \bar{t} b v_{e} e^{+}$, in Figure 5 we show the total cross section as a function of the electromagnetic form factors of the top quark $\widehat{a}_{V}$ and $\widehat{a}_{A}$ for $P_{e^{-}, e^{+}}=-80 \%, 0 \%$ [64], two different center-of-mass energies $\sqrt{s}=1.4,3 \mathrm{TeV}$ [17], and the Weizsacker-Williams photon virtuality $Q^{2}=2 \mathrm{GeV}^{2}$ [68-71] (Table 6). We can see from this figure that the total cross section changes strongly with $\sqrt{s}$ reaching $20 \%$ and $23 \%$ at the end of the range considered to $\widehat{a}_{V, A}$ with polarized and unpolarized beams.

In Figure 6 we present the limit contours for the dipole moments in the $\left(\widehat{a}_{V}-\widehat{a}_{A}\right)$ plane for the process $e^{+} e^{-} \rightarrow$ $e^{+} \gamma^{*} e^{-} \rightarrow \bar{t} b v_{e} e^{+}$. The curves are for $\sqrt{s}=1.4,3 \mathrm{TeV}$ and $\mathscr{L}=50,500,1500 \mathrm{fb}^{-1}$. We have used $Q^{2}=2 \mathrm{GeV}^{2}$ and $b$-tagging efficiency $=0.8$. 
TABLE 1: Bounds on the $\widehat{a}_{V}$ magnetic moment and $\widehat{a}_{A}$ electric dipole moment for the process $\gamma e^{-} \rightarrow \bar{t} b v_{e}(\gamma$ is the Compton backscattering photon) for $P_{e^{-}, e^{+}}=-80 \%, 0 \%, b$-tagging efficiency $=0.8, \delta_{\text {sys }}=0 \%, 5 \%$ at $2 \sigma$ and $3 \sigma$ C.L.

\begin{tabular}{|c|c|c|c|c|c|}
\hline$\sqrt{s}(\mathrm{TeV})$ & $\mathscr{L}\left(\mathrm{fb}^{-1}\right)$ & $\widehat{a}_{V}$ & $\left|\widehat{a}_{A}\right|$ & $\widehat{a}_{V}$ & $\left|\widehat{a}_{A}\right|$ \\
\hline \multicolumn{2}{|c|}{$2 \sigma$ C.L. } & \multicolumn{2}{|c|}{$\delta_{\text {sys }}=0 \%$} & \multicolumn{2}{|c|}{$\delta_{\text {sys }}=5 \%$} \\
\hline 1.4 & 50 & {$[-0.1091,0.1565]$} & 0.1615 & {$[-0.1902,0.2406]$} & 0.2352 \\
\hline 1.4 & 300 & {$[-0.0630,0.1103]$} & 0.1032 & {$[-0.1785,0.2289]$} & 0.2235 \\
\hline 1.4 & 500 & {$[-0.0534,0.1007]$} & 0.0908 & {$[-0.1775,0.2279]$} & 0.2224 \\
\hline 1.4 & 1000 & {$[-0.0424,0.0897]$} & 0.0763 & {$[-0.1767,0.2271]$} & 0.2217 \\
\hline 1.4 & 1500 & {$[-0.0369,0.0842]$} & 0.0689 & {$[-0.1765,0.2268]$} & 0.2214 \\
\hline 3 & 50 & {$[-0.0724,0.0816]$} & 0.0768 & {$[-0.1163,0.1254]$} & 0.1208 \\
\hline 3 & 300 & {$[-0.0447,0.0539]$} & 0.0491 & {$[-0.1120,0.1211]$} & 0.1164 \\
\hline 3 & 500 & {$[-0.0389,0.0480]$} & 0.0432 & {$[-0.1116,0.1207]$} & 0.1161 \\
\hline 3 & 1000 & {$[-0.0320,0.0412]$} & 0.0364 & {$[-0.1113,0.1204]$} & 0.1158 \\
\hline 3 & 1500 & {$[-0.0286,0.0377]$} & 0.0329 & {$[-0.1113,0.1203]$} & 0.1157 \\
\hline 3 & 2000 & {$[-0.0234,0.0325]$} & 0.0277 & {$[-0.1112,0.1203]$} & 0.1156 \\
\hline \multicolumn{2}{|c|}{$3 \sigma$ C.L. } & \multicolumn{2}{|c|}{$\delta_{\text {sys }}=0 \%$} & \multicolumn{2}{|c|}{$\delta_{\text {sys }}=5 \%$} \\
\hline$\overline{1.4}$ & 50 & {$[-0.1209,0.1683]$} & 0.1762 & {$[-0.2096,0.2599]$} & 0.2567 \\
\hline 1.4 & 300 & {$[-0.0703,0.1177]$} & 0.1126 & {$[-0.1968,0.2472]$} & 0.2438 \\
\hline 1.4 & 500 & {$[-0.0598,0.1071]$} & 0.0990 & {$[-0.1957,0.2460]$} & 0.2427 \\
\hline 1.4 & 1000 & {$[-0.0477,0.0950]$} & 0.0833 & {$[-0.1948,0.2452]$} & 0.2418 \\
\hline 1.4 & 1500 & {$[-0.0416,0.0889]$} & 0.0752 & {$[-0.1945,0.2449]$} & 0.2416 \\
\hline 3 & 50 & {$[-0.0794,0.0886]$} & 0.0838 & {$[-0.1273,0.1364]$} & 0.1318 \\
\hline 3 & 300 & {$[-0.0492,0.0583]$} & 0.0535 & {$[-0.1226,0.1317]$} & 0.1270 \\
\hline 3 & 500 & {$[-0.0428,0.0520]$} & 0.0472 & {$[-0.1222,0.1313]$} & 0.1266 \\
\hline 3 & 1000 & {$[-0.0353,0.0445]$} & 0.0396 & {$[-0.1219,0.1310]$} & 0.1263 \\
\hline 3 & 1500 & {$[-0.0315,0.0407]$} & 0.0358 & {$[-0.1218,0.1309]$} & 0.1262 \\
\hline 3 & 2000 & {$[-0.0258,0.0350]$} & 0.0301 & {$[-0.1217,0.1308]$} & 0.1261 \\
\hline
\end{tabular}

TABLE 2: Bounds on the $\widehat{a}_{V}$ magnetic moment and $\widehat{a}_{A}$ electric dipole moment for the process $\gamma e^{-} \rightarrow \bar{t} b v_{e}(\gamma$ is the Compton backscattering photon) for $b$-tagging efficiency $=0.8, \delta_{\text {sys }}=0 \%, 5 \%$ at $2 \sigma$ and $3 \sigma$ C.L.

\begin{tabular}{|c|c|c|c|c|c|}
\hline$\sqrt{s}(\mathrm{TeV})$ & $\mathscr{L}\left(\mathrm{fb}^{-1}\right)$ & $\widehat{a}_{V}$ & $\left|\widehat{a}_{A}\right|$ & $\widehat{a}_{V}$ & $\left|\widehat{a}_{A}\right|$ \\
\hline \multicolumn{2}{|c|}{$2 \sigma$ C.L. } & \multicolumn{2}{|c|}{$\delta_{\text {sys }}=0 \%$} & \multicolumn{2}{|c|}{$\delta_{\text {sys }}=5 \%$} \\
\hline 1.4 & 50 & {$[-0.1624,0.2158]$} & 0.1872 & {$[-0.2198,0.2733]$} & 0.2451 \\
\hline 1.4 & 300 & {$[-0.0958,0.1493]$} & 0.1196 & {$[-0.2003,0.2538]$} & 0.2255 \\
\hline 1.4 & 500 & {$[-0.0882,0.1353]$} & 0.1052 & {$[-0.1985,0.2520]$} & 0.2237 \\
\hline 1.4 & 1000 & {$[-0.0657,0.1192]$} & 0.0885 & {$[-0.1971,0.2506]$} & 0.2223 \\
\hline 1.4 & 1500 & {$[-0.0576,0.1111]$} & 0.0800 & {$[-0.1967,0.2501]$} & 0.2218 \\
\hline 3 & 50 & {$[-0.0845,0.0936]$} & 0.0887 & {$[-0.1201,0.1292]$} & 0.1246 \\
\hline 3 & 300 & {$[-0.0523,0.0614]$} & 0.0563 & {$[-0.1127,0.1218]$} & 0.1172 \\
\hline 3 & 500 & {$[-0.0454,0.0545]$} & 0.0494 & {$[-0.1120,0.1212]$} & 0.1165 \\
\hline 3 & 1000 & {$[-0.0375,0.0465]$} & 0.0414 & {$[-0.1115,0.1207]$} & 0.1160 \\
\hline 3 & 1500 & {$[-0.0334,0.0425]$} & 0.0373 & {$[-0.1114,0.1205]$} & 0.1159 \\
\hline 3 & 2000 & {$[-0.0273,0.0364]$} & 0.0312 & {$[-0.1113,0.1204]$} & 0.1158 \\
\hline \multicolumn{2}{|c|}{$3 \sigma$ C.L. } & \multicolumn{2}{|c|}{$\delta_{\text {sys }}=0 \%$} & \multicolumn{2}{|c|}{$\delta_{\text {sys }}=5 \%$} \\
\hline 1.4 & 50 & {$[-0.1793,0.2327]$} & 0.2043 & {$[-0.2420,0.2955]$} & 0.2674 \\
\hline 1.4 & 300 & {$[-0.1065,0.1599]$} & 0.1305 & {$[-0.2207,0.2742]$} & 0.2460 \\
\hline 1.4 & 500 & {$[-0.0912,0.1447]$} & 0.1149 & {$[-0.2188,0.2722]$} & 0.2440 \\
\hline 1.4 & 1000 & {$[-0.0735,0.1269]$} & 0.0969 & {$[-0.2172,0.2707]$} & 0.2425 \\
\hline 1.4 & 1500 & {$[-0.0645,0.1180]$} & 0.0873 & {$[-0.2167,0.2702]$} & 0.2420 \\
\hline 3 & 50 & {$[-0.0926,0.1017]$} & 0.0966 & {$[-0.1315,0.1406]$} & 0.1360 \\
\hline 3 & 300 & {$[-0.0574,0.0665]$} & 0.0616 & {$[-0.1234,0.1325]$} & 0.1279 \\
\hline 3 & 500 & {$[-0.0500,0.0591]$} & 0.0541 & {$[-0.1226,0.1318]$} & 0.1271 \\
\hline 3 & 1000 & {$[-0.0413,0.0504]$} & 0.0453 & {$[-0.1221,0.1312]$} & 0.1266 \\
\hline 3 & 1500 & {$[-0.0368,0.0459]$} & 0.0408 & {$[-0.1219,0.1310]$} & 0.1264 \\
\hline 3 & 2000 & {$[-0.0301,0.0392]$} & 0.0341 & {$[-0.1218,0.1309]$} & 0.1263 \\
\hline
\end{tabular}



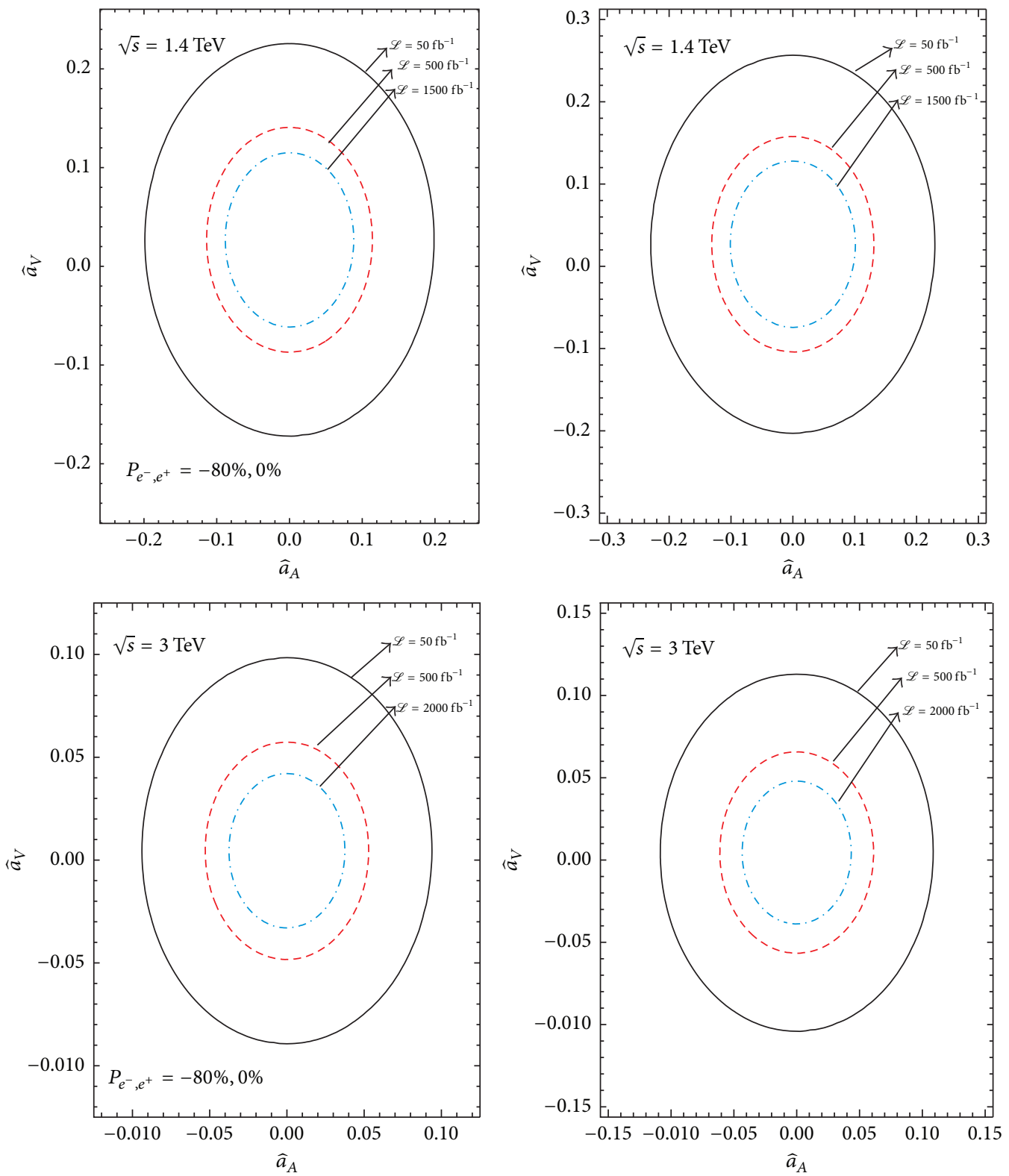

Figure 4: Limits contours at the 95\% C.L. in the $\widehat{a}_{V}-\widehat{a}_{A}$ plane for $\gamma e^{-} \rightarrow \bar{t} b v_{e}(\gamma$ is the Compton backscattering photon) and $\sqrt{s}=1.4,3$ TeV with polarized and unpolarized beams.

We summarize the bounds obtained on the anomalous parameters $\widehat{a}_{V}$ and $\widehat{a}_{A}$ for $b$-tagging efficiency $=0.8$, systematic uncertainties of $\delta_{\text {sys }}=0 \%, 5 \%, \sqrt{s}=1.4,3 \mathrm{TeV}, Q^{2}=$ $2 \mathrm{GeV}^{2}$, and $\mathscr{L}=50,300,500,1000,1500,2000 \mathrm{fb}^{-1}$ at $2 \sigma$ and $3 \sigma$ in Tables 4 and 5. The bounds obtained on these parameters with polarized/unpolarized beams are slightly moderate with respect to those obtained by the process $\gamma e^{-} \rightarrow \bar{t} b v_{e}$ as shown in Tables 1, 2, 3, and 4, respectively.

Finally, the predicted values of the corresponding production total cross sections of the process $e^{+} e^{-} \rightarrow e^{+} \gamma^{*} e^{-} \rightarrow$ $\bar{t} b v_{e} e^{+}$are listed in Table 4 as a function of $\widehat{a}_{V}$ and $\widehat{a}_{A}$ by assuming the initial electron (positron) beam polarization to be $-80 \%(0 \%)$ for $Q^{2}=2 \mathrm{GeV}^{2}, b$-tagging efficiency $=0.8$, and center-of-mass energies of CLIC - 1.4 TeV and CLIC $3 \mathrm{TeV}$.

It is worth mentioning that the ratio of the total cross section of the process $\gamma e^{-} \rightarrow \bar{t} b v_{e}$ ( $\gamma$ is the Compton backscattering photon) is generally about 18 times greater than the total cross section of the process $e^{+} e^{-} \rightarrow e^{+} \gamma^{*} e^{-} \rightarrow$ $\bar{t} b v_{e} e^{+}\left(\gamma^{*}\right.$ is the Weizsacker-Williams photon) and both total cross sections depend strongly on the dipole moments $\left(\widehat{a}_{V}\right.$ and $\left.\widehat{a}_{A}\right)$ and on the center-of-mass energy $(\sqrt{s})$ of the CLIC.

\section{Conclusions}

Although $\gamma e^{-}$and $\gamma \gamma$ processes require new detectors, $\gamma^{*} e^{-}$ and $\gamma^{*} \gamma^{*}$ are produced spontaneously at linear colliders 
TABLE 3: Total cross sections for the process $\gamma e^{-} \rightarrow \bar{t} b v_{e}\left(\gamma\right.$ is the Compton backscattering photon) as a function of $\widehat{a}_{V}$ and $\widehat{a}_{A}$ for $b$-tagging efficiency $=0.8$ at $P_{e^{-}, e^{+}}=-80 \%, 0 \%$ and $P_{e^{-}, e^{+}}=0 \%, 0 \%$, respectively.

\begin{tabular}{|c|c|c|c|c|}
\hline$\sqrt{s}(\mathrm{TeV})$ & $\widehat{a}_{V}$ & $\sigma_{\mathrm{Tot}}\left(\gamma e^{-} \rightarrow \bar{t} b v_{e}\right)(\mathrm{pb})$ & $\widehat{a}_{A}$ & $\sigma_{\mathrm{Tot}}\left(\gamma e^{-} \rightarrow \bar{t} b v_{e}\right)(\mathrm{pb})$ \\
\hline \multicolumn{5}{|c|}{$P_{e^{-}, e^{+}}=-80 \%, 0 \%$} \\
\hline 1.4 & -1 & $1.4328 \times 10^{-1}$ & -1 & $1.3862 \times 10^{-1}$ \\
\hline 1.4 & -0.5 & $7.5675 \times 10^{-2}$ & -0.5 & $7.3354 \times 10^{-2}$ \\
\hline 1.4 & 0 & $5.1582 \times 10^{-2}(\mathrm{SM})$ & 0 & $5.1582 \times 10^{-2}(\mathrm{SM})$ \\
\hline 1.4 & 0.5 & $7.1010 \times 10^{-2}$ & 0.5 & $7.3332 \times 10^{-2}$ \\
\hline 1.4 & 1 & $1.3393 \times 10^{-1}$ & 1 & $1.3862 \times 10^{-1}$ \\
\hline 3 & -1 & $5.4330 \times 10^{-1}$ & -1 & $5.3912 \times 10^{-1}$ \\
\hline 3 & -0.5 & $1.9331 \times 10^{-1}$ & -0.5 & $1.9120 \times 10^{-1}$ \\
\hline 3 & 0 & $7.5185 \times 10^{-2}(\mathrm{SM})$ & 0 & $7.5185 \times 10^{-2}(\mathrm{SM})$ \\
\hline 3 & 0.5 & $1.8907 \times 10^{-1}$ & 0.5 & $1.9117 \times 10^{-1}$ \\
\hline 3 & 1 & $5.3492 \times 10^{-1}$ & 1 & $5.3923 \times 10^{-1}$ \\
\hline \multicolumn{5}{|c|}{$P_{e^{-}, e^{+}}=0 \%, 0 \%$} \\
\hline 1.4 & -1 & $7.9603 \times 10^{-2}$ & -1 & $7.7002 \times 10^{-2}$ \\
\hline 1.4 & -0.5 & $4.2039 \times 10^{-2}$ & -0.5 & $4.0744 \times 10^{-2}$ \\
\hline 1.4 & 0 & $2.8659 \times 10^{-2}(\mathrm{SM})$ & 0 & $2.8659 \times 10^{-2}(\mathrm{SM})$ \\
\hline 1.4 & 0.5 & $3.9456 \times 10^{-2}$ & 0.5 & $4.0742 \times 10^{-2}$ \\
\hline 1.4 & 1 & $7.4405 \times 10^{-2}$ & 1 & $7.7019 \times 10^{-2}$ \\
\hline 3 & -1 & $3.0186 \times 10^{-1}$ & -1 & $2.9953 \times 10^{-1}$ \\
\hline 3 & -0.5 & $1.0738 \times 10^{-1}$ & -0.5 & $1.0621 \times 10^{-1}$ \\
\hline 3 & 0 & $4.1765 \times 10^{-2}(\mathrm{SM})$ & 0 & $4.1765 \times 10^{-2}(\mathrm{SM})$ \\
\hline 3 & 0.5 & $1.0502 \times 10^{-1}$ & 0.5 & $1.0620 \times 10^{-1}$ \\
\hline 3 & 1 & $2.9713 \times 10^{-1}$ & 1 & $2.9952 \times 10^{-1}$ \\
\hline
\end{tabular}

TABLE 4: Bounds on the $\widehat{a}_{V}$ magnetic moment and $\widehat{a}_{A}$ electric dipole moment for the process $e^{+} e^{-} \rightarrow e^{+} \gamma^{*} e^{-} \rightarrow \bar{t} b v_{e} e^{+}\left(\gamma^{*}\right.$ is the WeizsackerWilliams photon) for $Q^{2}=2 \mathrm{GeV}^{2}, P_{e^{-}, e^{+}}=-80 \%, 0 \%, b$-tagging efficiency $=0.8, \delta_{\text {sys }}=0 \%, 5 \%$ at $2 \sigma$ and $3 \sigma$ C.L.

\begin{tabular}{|c|c|c|c|c|c|}
\hline$\sqrt{s}(\mathrm{TeV})$ & $\mathscr{L}\left(\mathrm{fb}^{-1}\right)$ & $\widehat{a}_{V}$ & $\left|\widehat{a}_{A}\right|$ & $\widehat{a}_{V}$ & $\left|\widehat{a}_{A}\right|$ \\
\hline \multicolumn{2}{|c|}{$2 \sigma$ C.L. } & \multicolumn{2}{|c|}{$\delta_{\text {sys }}=0 \%$} & \multicolumn{2}{|c|}{$\delta_{\text {sys }}=5 \%$} \\
\hline 1.4 & 50 & {$[-0.3474,0.4397]$} & 0.3908 & {$[-0.3669,0.4592]$} & 0.4104 \\
\hline 1.4 & 300 & {$[-0.2078,0.3001]$} & 0.2497 & {$[-0.2647,0.3570]$} & 0.3074 \\
\hline 1.4 & 500 & {$[-0.1784,0.2707]$} & 0.2197 & {$[-0.2505,0.3428]$} & 0.2931 \\
\hline 1.4 & 1000 & {$[-0.1443,0.2366]$} & 0.1848 & {$[-0.2383,0.3306]$} & 0.2807 \\
\hline 1.4 & 1500 & {$[-0.1271,0.2194]$} & 0.1670 & {$[-0.2339,0.3262]$} & 0.2762 \\
\hline 3 & 50 & {$[-0.1806,0.2180]$} & 0.1984 & {$[-0.2037,0.2411]$} & 0.2216 \\
\hline 3 & 300 & {$[-0.1094,0.1469]$} & 0.1267 & {$[-0.1652,0.2026]$} & 0.1829 \\
\hline 3 & 500 & {$[-0.0944,0.1318]$} & 0.1115 & {$[-0.1608,0.1982]$} & 0.1785 \\
\hline 3 & 1000 & {$[-0.0769,0.1144]$} & 0.0937 & {$[-0.1573,0.1947]$} & 0.1750 \\
\hline 3 & 1500 & {$[-0.0681,0.1055]$} & 0.0847 & {$[-0.1561,0.1935]$} & 0.1738 \\
\hline 3 & 2000 & {$[-0.0547,0.0921]$} & 0.0713 & {$[-0.1555,0.1929]$} & 0.1732 \\
\hline \multicolumn{2}{|c|}{$3 \sigma$ C.L. } & \multicolumn{2}{|c|}{$\delta_{\text {sys }}=0 \%$} & \multicolumn{2}{|c|}{$\delta_{\text {sys }}=5 \%$} \\
\hline 1.4 & 50 & {$[-0.3827,0.4750]$} & 0.4264 & {$[-0.4040,0.4963]$} & 0.4478 \\
\hline 1.4 & 300 & {$[-0.2302,0.3225]$} & 0.2724 & {$[-0.2924,0.3847]$} & 0.3354 \\
\hline 1.4 & 500 & {$[-0.1980,0.2903]$} & 0.2397 & {$[-0.2769,0.3692]$} & 0.3197 \\
\hline 1.4 & 1000 & {$[-0.1607,0.2530]$} & 0.2016 & {$[-0.2636,0.3559]$} & 0.3063 \\
\hline 1.4 & 1500 & {$[-0.1418,0.2341]$} & 0.1822 & {$[-0.2587,0.3510]$} & 0.3013 \\
\hline 3 & 50 & {$[-0.1986,0.2360]$} & 0.2165 & {$[-0.2238,0.2612]$} & 0.2418 \\
\hline 3 & 300 & {$[-0.1208,0.1583]$} & 0.1383 & {$[-0.1817,0.2191]$} & 0.1995 \\
\hline 3 & 500 & {$[-0.1044,0.1419]$} & 0.1217 & {$[-0.1770,0.2144]$} & 0.1948 \\
\hline 3 & 1000 & {$[-0.0853,0.1228]$} & 0.1023 & {$[-0.1732,0.2106]$} & 0.1909 \\
\hline 3 & 1500 & {$[-0.0756,0.1131]$} & 0.0924 & {$[-0.1718,0.2092]$} & 0.1896 \\
\hline 3 & 2000 & {$[-0.0609,0.1081]$} & 0.0777 & {$[-0.1711,0.2086]$} & 0.1890 \\
\hline
\end{tabular}


TABLE 5: Bounds on the $\widehat{a}_{V}$ magnetic moment and $\widehat{a}_{A}$ electric dipole moment for the process $e^{+} e^{-} \rightarrow e^{+} \gamma^{*} e^{-} \rightarrow \bar{t} b v_{e} e^{+}\left(\gamma^{*}\right.$ is the WeizsackerWilliams photon) for $Q^{2}=2 \mathrm{GeV}^{2}, b$-tagging efficiency $=0.8, \delta_{\text {sys }}=0 \%, 5 \%$ at $2 \sigma$ and $3 \sigma$ C.L.

\begin{tabular}{|c|c|c|c|c|c|}
\hline$\sqrt{s}(\mathrm{TeV})$ & $\mathscr{L}\left(\mathrm{fb}^{-1}\right)$ & $\widehat{a}_{V}$ & $\left|\widehat{a}_{A}\right|$ & $\widehat{a}_{V}$ & $\left|\widehat{a}_{A}\right|$ \\
\hline \multicolumn{2}{|c|}{$2 \sigma$ C.L. } & \multicolumn{2}{|c|}{$\delta_{\text {sys }}=0 \%$} & \multicolumn{2}{|c|}{$\delta_{\text {sys }}=5 \%$} \\
\hline 1.4 & 50 & {$[-0.4088,0.5012]$} & 0.4527 & {$[-0.4219,0.5142]$} & 0.4657 \\
\hline 1.4 & 300 & {$[-0.2467,0.3390]$} & 0.2892 & {$[-0.2883,0.3806]$} & 0.3313 \\
\hline 1.4 & 500 & {$[-0.2126,0.0304]$} & 0.2545 & {$[-0.2673,0.3597]$} & 0.3101 \\
\hline 1.4 & 1000 & {$[-0.1728,0.2651]$} & 0.2140 & {$[-0.2482,0.3405]$} & 0.2907 \\
\hline 1.4 & 1500 & {$[-0.1527,0.2450]$} & 0.1934 & {$[-0.2409,0.3332]$} & 0.2833 \\
\hline 3 & 50 & {$[-0.2126,0.2493]$} & 0.2299 & {$[-0.2274,0.2654]$} & 0.2459 \\
\hline 3 & 300 & {$[-0.1300,0.1666]$} & 0.1468 & {$[-0.1726,0.2106]$} & 0.1908 \\
\hline 3 & 500 & {$[-0.1125,0.1491]$} & 0.1293 & {$[-0.1656,0.2035]$} & 0.1838 \\
\hline 3 & 1000 & {$[-0.0921,0.1287]$} & 0.1086 & {$[-0.1597,0.1977]$} & 0.1778 \\
\hline 3 & 1500 & {$[-0.0818,0.1184]$} & 0.0982 & {$[-0.1576,0.1956]$} & 0.1757 \\
\hline 3 & 2000 & {$[-0.0662,0.1028]$} & 0.0826 & {$[-0.1565,0.1945]$} & 0.1747 \\
\hline \multicolumn{2}{|c|}{$3 \sigma$ C.L. } & \multicolumn{2}{|c|}{$\delta_{\text {sys }}=0 \%$} & \multicolumn{2}{|c|}{$\delta_{\text {sys }}=5 \%$} \\
\hline 1.4 & 50 & {$[-0.4499,0.5422]$} & 0.4938 & {$[-0.4641,0.5554]$} & 0.5081 \\
\hline 1.4 & 300 & {$[-0.2727,0.3651]$} & 0.3155 & {$[-0.3182,0.4105]$} & 0.3614 \\
\hline 1.4 & 500 & {$[-0.2354,0.3277]$} & 0.2777 & {$[-0.2953,0.3876]$} & 0.3383 \\
\hline 1.4 & 1000 & {$[-0.1919,0.2842]$} & 0.2335 & {$[-0.2744,0.3667]$} & 0.3171 \\
\hline 1.4 & 1500 & {$[-0.1698,0.2622]$} & 0.2111 & {$[-0.2664,0.3587]$} & 0.3091 \\
\hline 3 & 50 & {$[-0.2335,0.2702]$} & 0.2508 & {$[-0.2497,0.2877]$} & 0.2682 \\
\hline 3 & 300 & {$[-0.1433,0.1799]$} & 0.1602 & {$[-0.1899,0.2279]$} & 0.2082 \\
\hline 3 & 500 & {$[-0.1242,0.1607]$} & 0.1410 & {$[-0.1822,0.2202]$} & 0.2005 \\
\hline 3 & 1000 & {$[-0.1019,0.1385]$} & 0.1185 & {$[-0.1758,0.2138]$} & 0.1940 \\
\hline 3 & 1500 & {$[-0.0906,0.1272]$} & 0.1071 & {$[-0.1735,0.2115]$} & 0.1917 \\
\hline 3 & 2000 & {$[-0.0736,0.1102]$} & 0.0901 & {$[-0.1723,0.2103]$} & 0.1906 \\
\hline
\end{tabular}

TABLE 6: Total cross sections for the process $e^{+} e^{-} \rightarrow e^{+} \gamma^{*} e^{-} \rightarrow \bar{t} b v_{e} e^{+}\left(\gamma^{*}\right.$ is the Weizsacker-Williams photon) as a function of $\widehat{a}_{V}$ and $\widehat{a}_{A}$ for $\mathrm{Q}^{2}=2 \mathrm{GeV}^{2}, b$ - tagging efficiency $=0.8$ at $P_{e^{-}, e^{+}}=-80 \%, 0 \%$ and $P_{e^{-}, e^{+}}=0 \%, 0 \%$, respectively.

\begin{tabular}{|c|c|c|c|c|}
\hline$\sqrt{s}(\mathrm{TeV})$ & $\widehat{a}_{V}$ & $\sigma_{\mathrm{Tot}}\left(e^{+} e^{-} \rightarrow e^{+} \gamma^{*} e^{-} \rightarrow \bar{t} b v_{e} e^{+}\right)(\mathrm{pb})$ & $\widehat{a}_{A}$ & $\sigma_{\mathrm{Tot}}\left(e^{+} e^{-} \rightarrow e^{+} \gamma^{*} e^{-} \rightarrow \bar{t} b v_{e} e^{+}\right)(\mathrm{pb})$ \\
\hline \multicolumn{5}{|c|}{$P_{e^{-}, e^{+}}=-80 \%, 0 \%$} \\
\hline 1.4 & -1 & $7.2429 \times 10^{-3}$ & -1 & $6.9011 \times 10^{-3}$ \\
\hline 1.4 & -0.5 & $4.2934 \times 10^{-3}$ & -0.5 & $4.1221 \times 10^{-3}$ \\
\hline 1.4 & 0 & $3.1962 \times 10^{-3}(\mathrm{SM})$ & 0 & $3.1962 \times 10^{-3}(\mathrm{SM})$ \\
\hline 1.4 & 0.5 & $3.9510 \times 10^{-3}$ & 0.5 & $4.1219 \times 10^{-3}$ \\
\hline 1.4 & 1 & $6.5593 \times 10^{-3}$ & 1 & $6.9011 \times 10^{-3}$ \\
\hline 3 & -1 & $3.2064 \times 10^{-2}$ & -1 & $3.1205 \times 10^{-2}$ \\
\hline 3 & -0.5 & $1.4380 \times 10^{-2}$ & -0.5 & $1.3952 \times 10^{-2}$ \\
\hline 3 & 0 & $8.1986 \times 10^{-3}(\mathrm{SM})$ & 0 & $8.1986 \times 10^{-3}(\mathrm{SM})$ \\
\hline 3 & 0.5 & $1.3521 \times 10^{-2}$ & 0.5 & $1.3951 \times 10^{-2}$ \\
\hline 3 & 1 & $3.0346 \times 10^{-2}$ & 1 & $3.1208 \times 10^{-2}$ \\
\hline \multicolumn{5}{|c|}{$P_{e^{-}, e^{+}}=0 \%, 0 \%$} \\
\hline 1.4 & -1 & $4.0240 \times 10^{-3}$ & -1 & $3.8340 \times 10^{-3}$ \\
\hline 1.4 & -0.5 & $2.3853 \times 10^{-3}$ & -0.5 & $2.2903 \times 10^{-3}$ \\
\hline 1.4 & 0 & $1.7754 \times 10^{-3}(\mathrm{SM})$ & 0 & $1.7754 \times 10^{-3}(\mathrm{SM})$ \\
\hline 1.4 & 0.5 & $2.1950 \times 10^{-3}$ & 0.5 & $2.2901 \times 10^{-3}$ \\
\hline 1.4 & 1 & $3.6438 \times 10^{-3}$ & 1 & $3.8338 \times 10^{-3}$ \\
\hline 3 & -1 & $1.7875 \times 10^{-2}$ & -1 & $1.7336 \times 10^{-2}$ \\
\hline 3 & -0.5 & $7.9900 \times 10^{-3}$ & -0.5 & $7.7515 \times 10^{-3}$ \\
\hline 3 & 0 & $4.5559 \times 10^{-3}(\mathrm{SM})$ & 0 & $4.5559 \times 10^{-3}(\mathrm{SM})$ \\
\hline 3 & 0.5 & $7.5127 \times 10^{-3}$ & 0.5 & $7.7508 \times 10^{-3}$ \\
\hline 3 & 1 & $1.6860 \times 10^{-2}$ & 1 & $1.7337 \times 10^{-2}$ \\
\hline
\end{tabular}



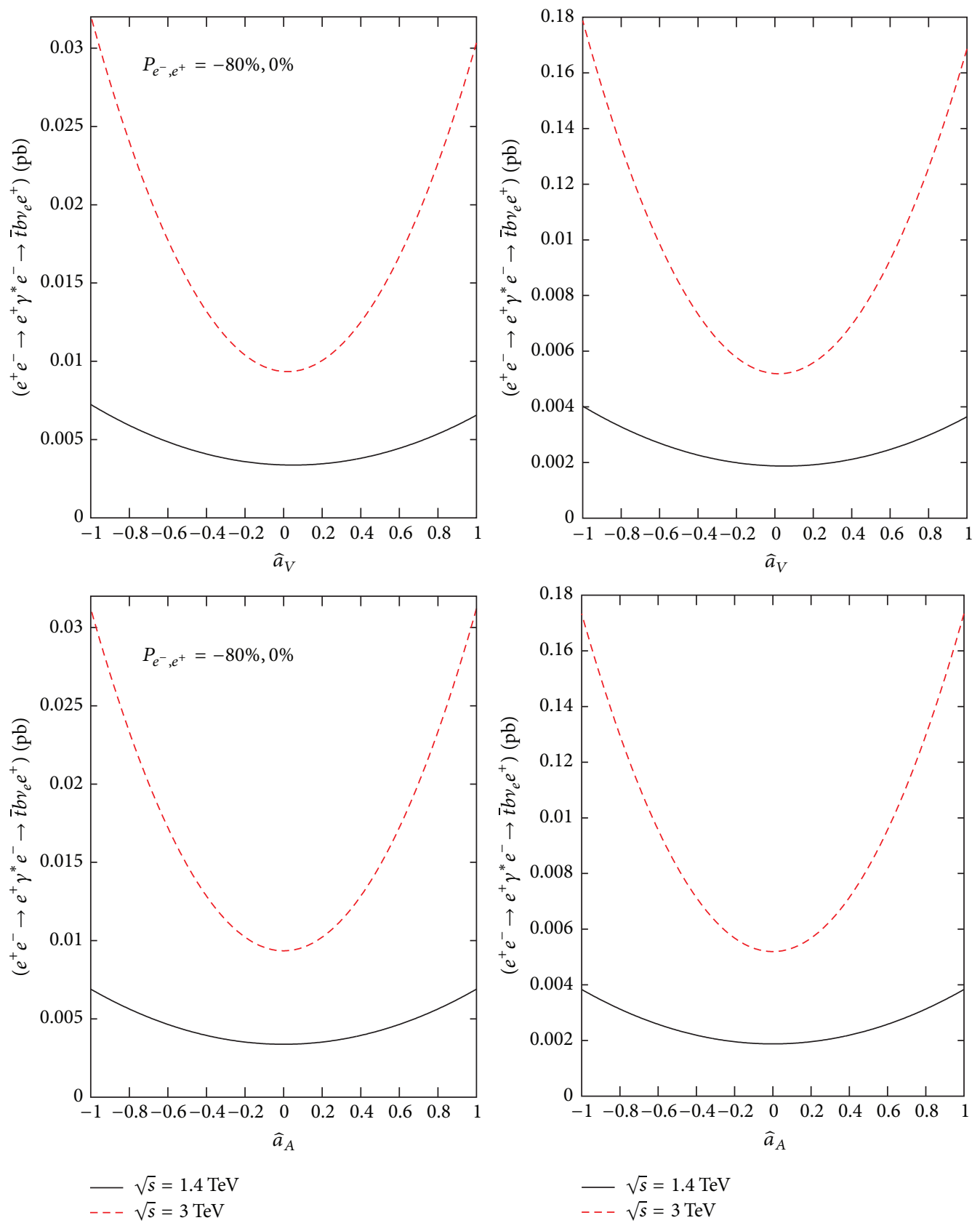

Figure 5: The integrated total cross section of the process $e^{+} e^{-} \rightarrow e^{+} \gamma^{*} e^{-} \rightarrow \bar{t} b v_{e} e^{+}\left(\gamma^{*}\right.$ is the Weizsacker-Williams photon) as a function of $\widehat{a}_{V}$ and $\widehat{a}_{A}$ with $\sqrt{s}=1.4,3 \mathrm{TeV}, Q^{2}=2 \mathrm{GeV}^{2}$ and polarized and unpolarized beams.

without any detectors. These processes will allow the future linear colliders to operate in two different modes, $\gamma^{*} e^{-}$and $\gamma^{*} \gamma^{*}$, opening up the opportunity for a wider search for new physics. Therefore, the $\gamma^{*} e^{-}$linear collisions represent an excellent opportunity to study top quark anomalous magnetic moment and electric dipole moment.

We have performed a study of the total cross section of the processes $\gamma e^{-} \rightarrow \bar{t} b v_{e}$ and $e^{+} e^{-} \rightarrow e^{+} \gamma^{*} e^{-} \rightarrow$ $\bar{t} b v_{e} e^{+}$, with polarized and unpolarized electron beams as a function of the anomalous couplings $\widehat{a}_{V}$ and $\widehat{a}_{A}$. We have also investigated anomalous $\widehat{a}_{V}$ and $\widehat{a}_{A}$ couplings for both polarized and unpolarized cases. The general behavior of the cross sections as a function of $\widehat{a}_{V}$ and $\widehat{a}_{A}$ couplings does not change. However, we can see from our calculations of the polarized and unpolarized cases that polarization increases the cross sections. The main reason for these results can be seen in Figure 2. There are four diagrams which contribute to the process and one of them includes the $t \bar{t} \gamma$ vertex. This diagram gives the maximum contribution to the total cross section. For $P_{e^{-}, e^{+}}=-80 \%, 0 \%$, this contribution is dominant due to the structure of the $W e^{-} v_{e}$ vertex. We can appreciate from these figures that lepton polarization can improve the 

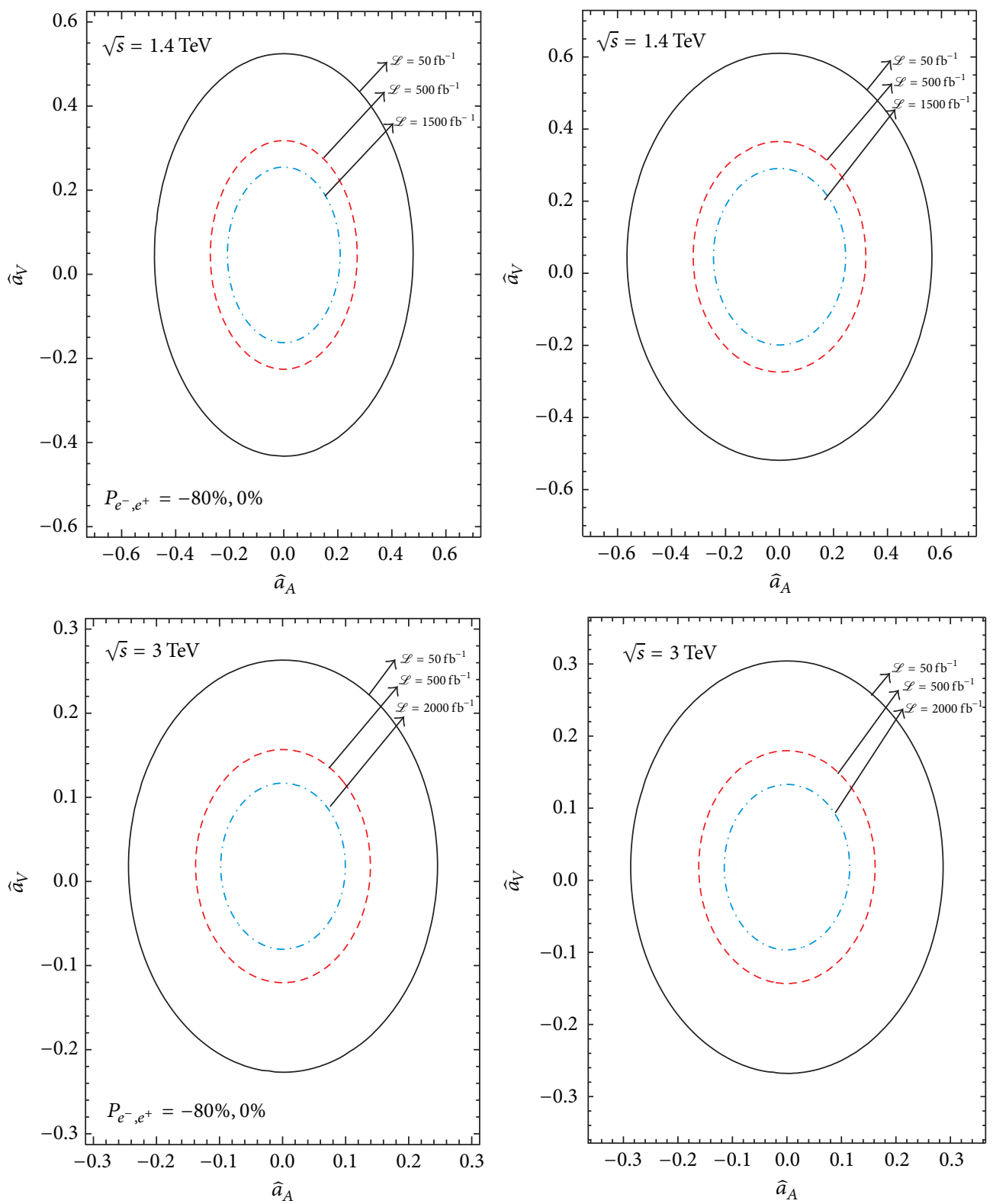

Figure 6: Limits contours at the 95\% C.L. in the $\widehat{a}_{V}-\widehat{a}_{A}$ plane for $e^{+} e^{-} \rightarrow e^{+} \gamma^{*} e^{-} \rightarrow \bar{t} b v_{e} e^{+}\left(\gamma^{*}\right.$ is the Weizsacker-Williams photon) with $\sqrt{s}=1.4,3 \mathrm{TeV}, Q^{2}=2 \mathrm{GeV}^{2}$ and polarized and unpolarized beams.

bounds on the anomalous couplings. The analysis is shown in Figures 3 and 5 with Compton backscattering photon and Weizsacker-Williams photon virtuality of $Q^{2}=2 \mathrm{GeV}^{2}$ and $b$-tagging efficiency $=0.8$. In both processes, the cross section shows a strong dependence on the anomalous couplings $\widehat{a}_{V}$ and $\widehat{a}_{A}$, as well as on the center-of-mass energy $\sqrt{s}$. This variation of the cross section for $\sqrt{s}=1.4,3 \mathrm{TeV}$ is of the order $24.5 \%, 26.6 \%$ and $20 \%, 23 \%$ for $\gamma e^{-} \rightarrow \bar{t} b v_{e}$ and $e^{+} e^{-} \rightarrow e^{+} \gamma^{*} e^{-} \rightarrow \bar{t} b v_{e} e^{+}$, respectively.

We also include contour plots for the dipole moments at the $95 \%$ C.L. in the $\left(\widehat{a}_{V}-\widehat{a}_{A}\right)$ plane for the processes $\gamma e^{-} \rightarrow \bar{t} b v_{e}$ and $e^{+} e^{-} \rightarrow e^{+} \gamma^{*} e^{-} \rightarrow \bar{t} b v_{e} e^{+}$for $Q^{2}=2 \mathrm{GeV}^{2}$ and $\sqrt{s}=1.4,3 \mathrm{TeV}$ in Figures 4 and 6 . The contours are consistent with the results obtained in Tables 1, 2, 4, and 5. The bounds obtained in these tables are competitive with those recently reported in the literature [54-57] and we can observe a strong correlation between the center-of-mass energy $\sqrt{s}$, integrated luminosity $\mathscr{L}$, and the dipole moments $\widehat{a}_{V}$ and $\widehat{a}_{A}$.

Other promising production modes for studying the cross section and the electromagnetic dipole moments $\widehat{a}_{V}$ and $\widehat{a}_{A}$ of the top quark are the processes $\gamma \gamma \rightarrow t \bar{t}$ (Compton backscattering photon), $\gamma^{*} \gamma^{*} \rightarrow t \bar{t}$ (Weizsacker-Williams photon), and $\gamma \gamma^{*} \rightarrow t \bar{t}$ (Compton backscattering photon, Weizsacker-Williams photon), respectively. These processes are one of the most important sources of $t \bar{t}$ pair production and represent new physics effects at a high-energy and highluminosity linear electron positron collider as the CLIC.

In conclusion, we have found that the processes $\gamma e^{-} \rightarrow$ $\bar{t} b v_{e}$ and $e^{+} e^{-} \rightarrow e^{+} \gamma^{*} e^{-} \rightarrow \bar{t} b v_{e} e^{+}$in the $\gamma e^{-}$and $\gamma^{*} e^{-}$collision modes at the high energies and luminosities 
expected at the CLIC can be used as a probe to bound the magnetic moment $\widehat{a}_{V}$ and electric dipole moment $\widehat{a}_{A}$ of the top quark. In particular, using integrated luminosity $2 a b^{-1}$, center-of-mass energies of $3 \mathrm{TeV}$, and $b$-tagging efficiency $=$ 0.8 and considering the systematic uncertainty $\delta_{\text {sys }}=5 \%$, we derive bounds on the dipole moments of the top quark at $2 \sigma$ and $3 \sigma(90 \%$ and 95\%) C.L.: $-0.1112(-0.1217) \leq$ $\widehat{a}_{V} \leq 0.1203(0.1308),\left|\widehat{a}_{A}\right|=0.1156(0.1261)$ and $-0.1113(-0.1218) \leq \widehat{a}_{V} \leq 0.1204$ (0.1309), $\left|\widehat{a}_{A}\right|=$ $0.1158(0.1263)$ for $\gamma e^{-} \rightarrow \bar{t} b v_{e}$ with unpolarized and polarized $e^{-}$beams. For $e^{+} e^{-} \rightarrow e^{+} \gamma^{*} e^{-} \rightarrow \bar{t} b v_{e} e^{+}$with polarized and unpolarized electron beams, $-0.1555(-0.1711) \leq$ $\widehat{a}_{V} \leq 0.1929(0.2086),\left|\widehat{a}_{A}\right|=0.1732(0.1890)$ and $-0.1565(-0.1723) \leq \widehat{a}_{V} \leq 0.1945(0.2103),\left|\widehat{a}_{A}\right|=$ $0.1747(0.1906)$. These results are competitive with those recently reported in previous studies [54-57]. To our knowledge, our numerical results for the dipole moments of the top quark through the single top production processes have never been reported in the literature before and could be of relevance for the scientific community.

\section{Competing Interests}

The authors declare that there is no conflict of interests regarding the publication of this paper.

\section{Acknowledgments}

A. Gutiérrez-Rodríguez acknowledges support from CONACyT, SNI, and PROFOCIE (México).

\section{References}

[1] S. L. Glashow, "Partial-symmetries of weak interactions," Nuclear Physics, vol. 22, no. 4, pp. 579-588, 1961.

[2] S. Weinberg, "A model of leptons," Physical Review Letters, vol. 19, no. 21, pp. 1264-1266, 1967.

[3] A. Salam, Elementary Particle Theory, Edited by N. Svartholm, Almquist and Wiskell, Stockholm, Sweden, 1968.

[4] K. A. Olive, K. Agashe, C. Amsler et al., "Review of particle physics," Chinese Physics C, vol. 38, no. 9, Article ID 090001, 2014.

[5] ATLAS Collaboration, "Measurement of the inclusive $t \bar{t} \gamma$ cross section with the ATLAS detector" Tech. Rep. ATLAS-CONF2011-153, ATLAS-COM-CONF-2011-186, 2011.

[6] CMS Collaboration, "Measurement of the inclusive top-quark pair + photon production cross section in the muon + jets channel in pp collisions at $8 \mathrm{TeV}$," Tech. Rep. CMS-PAS-TOP13011, 2014.

[7] T. Abe, N. Arkani-Hamed, D. Asner et al., "Linear collider physics resource book for snowmass 2001-part 1: introduction," https://arxiv.org/abs/hep-ex/0106055.

[8] T. Abe, N. Arkani-Hamed, D. Asner et al., "Linear collider physics resource book for snowmass 2001-part 2: higgs and supersymmetry studies," https://arxiv.org/abs/hep-ex/0106056.

[9] G. Aarons, T. Abe, J. Abernathy et al., "International Linear Collider Reference Design Report Volume 2: PHYSICS AT THE ILC," https://arxiv.org/abs/0709.1893.

[10] J. Brau, Y. Okada, N. Walker et al., "ILC reference design report volume 1-executive summary," https://arxiv.org/abs/0712.1950.
[11] H. Baer, T. Barklow, K. Fujii et al., "The international linear collider technical design report-volume 2: physics," https://arxiv .org/abs/1306.6352.

[12] E. Accomando, A. Aranda, E. Ateser et al., "Physics at the CLIC Multi-TeV Linear Collider," https://arxiv.org/abs/hep-ph/ 0412251.

[13] D. Dannheim, P. Lebrun, L. Linssen et al., "CLIC e+e- linear collider studies," https://arxiv.org/abs/1208.1402.

[14] J. A. Aguilar-Saavedra, J. Alcaraz, A. Ali et al., "TESLA: the superconducting electron positron linear collider with an integrated $\mathrm{x}$-ray laser laboratory. Technical design report. Part III. Physics at an $\mathrm{e}^{+} \mathrm{e}^{-}$linear collider," https://arxiv.org/abs/hep-ph/ 0106315.

[15] T. Abe, N. Arkani-Hamed, D. Asner et al., "Linear collider physics resource book for snowmass 2001-part 3: studies of exotic and standard model physics," https://arxiv.org/abs/hepex/0106057.

[16] T. Abe, N. Arkani-Hamed, D. Asner et al., "Linear collider physics resource book for snowmass 2001-part 4: theoretical, accelerator, and experimental options," https://arxiv.org/abs/ hep-ex/0106058.

[17] H. Abramowicz, A. Abusleme, K. Afanaciev et al., "Physics at the CLIC e+e- linear collider-input to the snowmass process 2013," https://arxiv.org/abs/1307.5288.

[18] A. Soni and R. M. Xu, "Electric dipole moment of the top quark in Higgs-boson-exchange models of CP nonconservation," Physical Review Letters, vol. 69, no. 1, article 33, 1992.

[19] A. Soni and R. M. Xu, "Analysis for magnetic moment and electric dipole moment form factors of the top quark via $e^{+} e^{-} \rightarrow$ tt $t^{-}$, Physical Review D, vol. 45, no. 7, p. 2405, 1992.

[20] A. Bartl, E. Christova, T. Gajdosik, and W. Majerotto, "Electroweak dipole moment form factors of the top quark in supersymmetry," Nuclear Physics B-Proceedings Supplements, vol. 66, no. 1-3, pp. 75-78, 1998.

[21] W. Hollik, J. I. Illana, S. Rigolin, C. Schappacher, and D. Stöckinger, "Top dipole form-factors and loop induced CP violation in supersymmetry," Nuclear Physics B, vol. 551, no. 3, 1999, Erratum to Nuclear Physics B, vol. 557, p. 407, 1999.

[22] C. S. Huang and T. J. Li, "Electric dipole moment and chromoelectric electric dipole moment of the top quark in $S U(3)_{C} \times$ $S U(3)_{L} \times U(1)_{X}$ model," Zeitschrift für Physik, vol. 68, no. 2, pp. 319-324, 1995.

[23] G. A. González-Sprinberg, R. Martínez, and J. Vidal, “Top quark tensor couplings," Journal of High Energy Physics, vol. 1107, p. 94, 2011.

[24] R. Martínez, J. Alexis Rodríguez, and M. Vargas, "Constraint on the magnetic moment of the top quark," Physical Review D, vol. 60, no. 7, Article ID 077504, 1999.

[25] F. Larios, M. A. Pérez, and C. P. Yuan, "Analysis of $t-b-W$ and $t-t-Z$ couplings from CLEO and LEP/SLC data," Physics Letters $B$, vol. 457, no. 4, pp. 334-340, 1999.

[26] D. Atwood, A. Aeppli, and A. Soni, "Extracting anomalous gluona top-quark effective couplings at the supercolliders," Physical Review Letters, vol. 69, no. 19, p. 2754, 1992.

[27] P. Poulose and S. D. Rindani, "Electric dipole moment of the top quark and CP-violating asymmetries in $\gamma \vec{\gamma} t \bar{t}$," Physical Review $D$, vol. 57, no. 9, Article ID 5444, 1998, Erratum: Physical Review D, vol. 61, Article ID 119902, 2000.

[28] S. Y. Choi and K. Hagiwara, "Probing the top-quark electric dipole moment at a photon linear collider," Physics Letters B, vol. 359, no. 3-4, pp. 369-374, 1995. 
[29] P. Poulose and S. D. Rindani, "Improved bounds on the dipole moments of the tau neutrino from high-energy $\gamma^{*} e^{-}$and $\gamma^{*} \gamma^{*}$ collisions at the ILC and CLIC," Physical Review D, vol. 91, no. 9, Article ID 093008, 2015.

[30] E. Boos, M. Dubinin, A. Pukhov, M. Sachwitz, and H. J. Schreiber, "Single top production in $\mathrm{e}^{+} \mathrm{e}^{-}, \mathrm{e}^{-} \mathrm{e}^{-}$, gamma e and gamma gamma collisions," The European Physical Journal C, vol. 21, p. 81, 2001.

[31] P. Batra and T. M. P. Tait, "Measuring the $W-t-b$ interaction at the ILC," Physical Review D, vol. 74, no. 5, Article ID 054021, 6 pages, 2006.

[32] J. Fuster, I. García, P. Gomis, M. Perelló, E. Ros, and M. Vos, "Study of single top production at high energy electron positron colliders," The European Physical Journal C, vol. 75, article 223, 2015.

[33] E. Boos and L. Dudko, "The single top quark physics," International Journal of Modern Physics A, vol. 27, no. 26, Article ID 1230026, 2012.

[34] E. Boos, “Top quarks at photon colliders," Nuclear Instruments and Methods in Physics Research Section A: Accelerators, Spectrometers, Detectors and Associated Equipment, vol. 472, no. 1-2, pp. 22-29, 2001.

[35] E. Boos, A. Pukhov, M. Sachwitz, and H. J. Schreiber, "Probing anomalous Wtb coupling via single top production at $\mathrm{TeV}$ energy ye colliders," Physics Letters B, vol. 404, no. 1-2, pp. 119123, 1997.

[36] G. Jikia, "Single t-quark production in high-energy $\gamma$ e collisions," Nuclear Physics B, vol. 374, no. 1, pp. 83-98, 1992.

[37] E. Boos, Y. Kurihara, M. Sachwitz, H. J. Schreiber, S. Shichanin, and Y. Shimizu, "Top quark production in the reaction $\mathrm{e}^{+} \mathrm{e}^{-} \rightarrow$ evtb at linear collider energies," Zeitschrift für Physik C, vol. 70, no. 2, pp. 255-261, 1996.

[38] E. Boos, A. Pukhov, M. Sachwitz, and H. J. Schreiber, "Higgs and top production in the reaction $\gamma e \rightarrow v b \bar{b} W$," Zeitschrift für Physik C, vol. 75, no. 2, pp. 237-244, 1997.

[39] J.-J. Cao, J.-X. Wang, J. M. Yang, B.-L. Young, and X. Zhang, "Probing anomalous top quark couplings at ey colliders," Physical Review D, vol. 58, no. 9, Article ID 094004, 7 pages, 1998.

[40] G. Weiglein, T. Barklow, E. Boos et al., "Physics interplay of the LHC and the ILC," Physics Reports, vol. 426, no. 2-6, pp. 47-358, 2006.

[41] A. P. Heinson, A. S. Belyaev, and E. E. Boos, "Single top quarks at the Fermilab Tevatron," Physical Review D, vol. 56, no. 5, pp. 3114-3128, 1997.

[42] D. O. Carlson and C.-P. Yuan, "Studying the top quark via the $W$-gluon fusion process," Physics Letters B, vol. 306, no. 3-4, pp. 386-390, 1993.

[43] D. O. Carlson, E. Malkawi, and C. P. Yuan, "Probing the couplings of the top quark to gauge bosons," Physics Letters B, vol. 337, no. 1-2, pp. 145-151, 1994.

[44] E. H. Simmons, "New gauge interactions and single top-quark production," Physical Review D, vol. 55, no. 9, pp. 5494-5500, 1997.

[45] D. Atwood, S. Bar-Shalom, G. Eilam, and A. Soni, "CP nonconservation in $p \bar{p} \rightarrow t \bar{b} X$ at the Fermilab Tevatron," Physical Review D, vol. 54, no. 9, pp. 5412-5416, 1996.

[46] C. S. Li, R. J. Oakes, and J. M. Yang, "Yukawa corrections to single top-quark production at the Fermilab Tevatron in the two-Higgs-doublet models," Physical Review D, vol. 55, no. 3, p. $1672,1997$.
[47] E. Malkawi and T. Tait, "Top-quark-charm-quark strong flavorchanging neutral currents at the Fermilab Tevatron," Physical Review D, vol. 54, no. 9, pp. 5758-5762, 1996.

[48] A. Datta, J. M. Yang, B. Young, and X. Zhang, " $R$-parityviolating supersymmetry effects and signals in single top quark production at the Fermilab Tevatron," Physical Review D, vol. 56, no. 5, pp. 3107-3113, 1997.

[49] R. J. Oakes, K. Whisnant, J. M. Yang, B. Young, and X. Zhang, "Single top quark production as a probe of $R$-parity-violating supersymmetry at pp and $p \bar{p}$ colliders," Physical Review D, vol. 57, no. 1, pp. 534-540, 1998.

[50] W. Bernreuther, R. Bonciani, T. Gehrmann et al., "QCD corrections to static heavy-quark form factors," Physical Review Letters, vol. 95, no. 26, Article ID 261802, 2005.

[51] F. Hoogeveen, "The standard model prediction for the electric dipole moment of the electron," Nuclear Physics B, vol. 341, no. 2, pp. 322-340, 1990.

[52] M. E. Pospelov and I. B. Khriplovich, "Electric dipole moment of the $\mathrm{W}$ boson and the electron in the Kobayashi-Maskawa model," Soviet Journal of Nuclear Physics, vol. 53, pp. 638-640, 1991, Yadernaya Fizika, vol. 53, pp. 1030-1033, 1991.

[53] T. Ibrahim and P. Nath, “Top quark electric dipole moment in a minimal supersymmetric standard model extension with vectorlike multiplets," Physical Review D, vol. 82, no. 5, Article ID 055001, 10 pages, 2010.

[54] U. Baur, A. Juste, L. H. Orr, and D. Rainwater, "Probing electroweak top quark couplings at hadron colliders," Physical Review D, vol. 71, no. 5, Article ID 054013, 2005.

[55] A. O. Bouzas and F. Larios, "Electromagnetic dipole moments of the top quark," Physical Review D, vol. 87, no. 7, Article ID 074015, 7 pages, 2013.

[56] A. O. Bouzas and F. Larios, "Probing $t t \gamma$ and $t t Z$ couplings at the LHeC," Physical Review D, vol. 88, no. 9, Article ID 094007, 2013.

[57] S. Fayazbakhsh, S. T. Monfared, and M. M. Najafabadi, “Top quark anomalous electromagnetic couplings in photon-photon scattering at the LHC," Physical Review D, vol. 92, no. 1, Article ID 014006, 2015.

[58] M. S. Amjad, M. Boronat, T. Frisson et al., "A precise determination of top quark electro-weak couplings at the ILC operating at $\sqrt{s}=500 \mathrm{GeV}$," https://arxiv.org/abs/1307.8102.

[59] A. Juste, Y. Kiyo, F. Petriello et al., "Report of the 2005 Snowmass Top/QCD Working Group," https://arxiv.org/abs/hep-ph/ 0601112.

[60] D. Asner, A. Hoang, Y. Kiyo, R. Pöschl, Y. Sumino, and M. Vos, "Top quark precision physics at the International Linear Collider," https://arxiv.org/abs/1307.8265.

[61] J. F. Kamenik, M. Papucci, and A. Weiler, "Constraining the dipole moments of the top quark," Physical Review D, vol. 85, no. 7, Article ID 071501, 2012.

[62] J. A. Aguilar-Saavedra, "A minimal set of top anomalous couplings," Nuclear Physics B, vol. 812, no. 1-2, pp. 181-204, 2009.

[63] J. A. Aguilar-Saavedra, M. C. N. Fiolhais, and A. Onofre, "Top effective operators at the ILC," Journal of High Energy Physics, vol. 2012, article 180, 2012.

[64] G. Moortgat-Pick, T. Abe, G. Alexander et al., "Polarized positrons and electrons at the linear collider," Physics Reports, vol. 460, no. 4-5, pp. 131-243, 2008.

[65] V. M. Budnev, I. F. Ginzburg, G. V. Meledin, and V. G. Serbo, "The two-photon particle production mechanism. Physical problems. Applications. Equivalent photon approximation," Physics Reports, vol. 15, no. 4, pp. 181-282, 1975. 
[66] G. Baur, K. Hencken, D. Trautmann, S. Sadovsky, and Y. Kharlov, "Coherent $\gamma \gamma$ and $\gamma A$ interactions in very peripheral collisions at relativistic ion colliders," Physics Reports, vol. 364, no. 5, pp. 359-450, 2002.

[67] K. Piotrzkowski, "Tagging two-photon production at the CERN Large Hadron Collider," Physical Review D, vol. 63, no. 7, Article ID 071502, 2001.

[68] M. Acciarri, O. Adrianip, M. Aguilar-Benitez et al., "Measurement of the anomalous magnetic and electric dipole moments of the tau lepton," Physics Letters B, vol. 434, no. 1-2, pp. 169-179, 1998.

[69] K. Ackerstaff, G. Alexanderv, J. Allisonp et al., "An upper limit on the anomalous magnetic moment of the $\tau$ lepton," Physics Letters B, vol. 431, no. 1-2, pp. 188-198, 1998.

[70] I. Şahin, "Electromagnetic properties of the neutrinos in $\gamma p$ collision at the LHC," Physical Review D, vol. 85, no. 3, Article ID 033002, 8 pages, 2012.

[71] A. A. Billur, M. Köksal et al., "Probe of the electromagnetic moments of the tau lepton in gamma-gamma collisions at the CLIC," Physical Review D, vol. 89, no. 3, Article ID 037301, 2014.

[72] A. Abulencia, J. Adelman, T. Affolder et al., "Observation of exclusive electron-positron production in hadron-hadron collisions," Physical Review Letters, vol. 98, no. 11, Article ID 112001, 7 pages, 2007.

[73] T. Aaltonen, J. Adelman, T. Akimoto et al., "Search for exclusive $Z$-boson production and observation of high-mass $p \bar{p} \rightarrow$ $p \gamma \gamma \bar{p} \rightarrow p l^{+} l^{-} \bar{p}$ Events in $p \bar{p}$ Collisions at $\sqrt{s}=1.96 \mathrm{TeV}, "$ Physical Review Letters, vol. 102, no. 22, Article ID 222002, 2009.

[74] T. Aaltonen, P. Mehtala, R. Orava et al., "Observation of Exclusive Charmonium Production and $\gamma \gamma \rightarrow \mu^{+} \mu^{-}$in $p \bar{p}$ Collisions at $\sqrt{s}=1.96 \mathrm{TeV}$," Physical Review Letters, vol. 102, no. 24, Article ID 242001, 2009.

[75] S. Chatrchyan, V. Khachatryan, A. M. Sirunyan et al., "Exclusive $\gamma \gamma \rightarrow \mu^{+} \mu^{-}$production in proton-proton collisions at $\sqrt{s}=$ $7 \mathrm{TeV}$," Journal of High Energy Physics, vol. 2012, no. 1, article 052, 2012.

[76] S. Chatrchyan, V. Khachatryan, A. M. Sirunyan et al., "Search for exclusive or semi-exclusive $\gamma \gamma$ production and observation of exclusive and semi-exclusive $e^{+} e^{-}$production in pp collisions at $\sqrt{s}=7 \mathrm{TeV}$," Journal of High Energy Physics, vol. 2012, artice 80, 2012.

[77] V. M. Abazov, M. J. Wetstein, T. Adams et al., "Search for anomalous quartic $W W \gamma \gamma$ couplings in dielectron and missing energy final states in $p \bar{p}$ collisions at $\sqrt{s}=1.96 \mathrm{TeV}$," Physical Review D, vol. 88, no. 1, Article ID 012005, 2013.

[78] S. Chatrchyan, V. Khachatryan, A. M. Sirunyan et al., "Study of exclusive two-photon production of $\mathrm{W}^{+} \mathrm{W}^{-}$in pp collisions at $\sqrt{s}=7 \mathrm{TeV}$ and constraints on anomalous quartic gauge couplings," Journal of High Energy Physics, vol. 2013, no. 7, article 116, 2013.

[79] S. C. İnan, "Exclusive excited leptons search in two lepton final states at the CERN LHC," Physical Review D, vol. 81, no. 11, Article ID 115002, 7 pages, 2010.

[80] S. C. Inan, "Dimension-six anomalous tq $\gamma$ couplings in $\gamma \gamma$ collision at the LHC," Nuclear Physics B, vol. 897, pp. 289-301, 2015.

[81] S. C. İnan, "Fourth generation leptons search in $\gamma \gamma \rightarrow \ell^{-} \ell^{+}$ process at the CERN-LHC," International Journal of Modern Physics A, vol. 26, no. 21, pp. 3605-3613, 2011.
[82] I. Sahin and S. C. Inan, "Probe of unparticles at the LHC in exclusive two lepton and two photon production via photonphoton fusion," Journal of High Energy Physics, vol. 2009, article 9, 2009.

[83] S. Atağ, S. C. İnan, and İ. Sahin, "Extra dimensions in $\gamma \gamma \rightarrow \gamma \gamma$ process at the CERN-LHC," Journal of High Energy Physics, vol. 2010, article 42, 2010.

[84] I. Sahin and B. Sahin, "Anomalous quartic $Z Z \gamma \gamma$ couplings in $\gamma p$ collision at the LHC," Physical Review D, vol. 86, no. 11, Article ID 115001, 2012.

[85] B. Sahin and A. A. Billur, "Anomalous Wtb couplings in $\gamma p$ collision at the LHC," Physical Review D, vol. 86, no. 7, Article ID 074026, 2012.

[86] A. Senol, "Anomalous quartic WW $\gamma \gamma$ and ZZ $\gamma \gamma$ couplings in $\gamma \mathrm{p}$ collision at the LHC," International Journal of Modern Physics A, vol. 29, no. 26, Article ID 1450148, 10 pages, 2014.

[87] A. Senol, " $Z Z \gamma$ and $Z \gamma \gamma$ anomalous coupling in $\gamma p$ collision at the LHC," Physical Review D, vol. 87, Article ID 073003, 2013.

[88] S. Fichet, G. von Gersdorff, B. Lenzi, C. Royon, and M. Saimpert, "Light-by-light scattering with intact protons at the LHC: from Standard model to New Physics," Journal of High Energy Physics, vol. 1502, p. 165, 2015.

[89] H. Sun, "Dark matter searches in jet plus missing energy events in $\gamma p$ collisions at the CERN LHC," Physical Review D, vol. 90, no. 3, Article ID 035018, 12 pages, 2014.

[90] H. Sun, "Probe anomalous tqy couplings through single top photoproduction at the LHC", Nuclear Physics B, vol. 886, pp. 691-711, 2014.

[91] H. Sun, Y. J. Zhou, and H. S. Hou, "NLO QCD corrections to Single Top and W associated photoproduction at the LHC with forward detector acceptances," Journal of High Energy Physics, vol. 2015, no. 2, article 64, 2015.

[92] A. Senol and M. Koksal, "Probe of anomalous quartic WWZ $\gamma$ couplings in photon-photon collisions," Journal of High Energy Physics, vol. 1503, p. 139, 2015.

[93] S. Atağ and A. A. Billur, "Possibility of determining $\tau$ lepton electromagnetic moments in $\gamma \gamma \rightarrow \tau^{+} \tau^{-}$process at the CERNLHC," Journal of High Energy Physics, vol. 2010, no. 11, article 060, 2010.

[94] A. Belyaev, N. D. Christensen, and A. Pukhov, "CalcHEP 3.4 for collider physics within and beyond the Standard Model," Computer Physics Communications, vol. 184, no. 7, pp. 17291769, 2013.

[95] G. Aad, B. Abbott, J. Abdallah et al., "Measurement of the $\mathrm{t}$-channel single top-quark production cross section in $\mathrm{pp}$ collisions at with the ATLAS detector," Physics Letters B, vol. 717, no. 4, pp. 330-350, 2012.

[96] G. Abbiendi, C. Ainsleye, P. F. Åkesson et al., "Search for single top quark production at LEP2," Physics Letters B, vol. 521, no. 3-4, pp. 181-194, 2001.

[97] M. Martinez and R. Miquel, "Multiparameter fits to the $t$ anti$\mathrm{t}$ threshold observables at a future e+e- collider," The European Physical Journal, vol. 27, no. 1, pp. 49-55, 2003.

[98] A. Juste, M. Martinez, and D. Schulte, “Top threshold studies," in Proceedings of the Joint ECFA/DESY Study: Physics and Detectors Conference, R. Settles, Ed., vol. C96-11-20, pp. 19-30, 1997. 

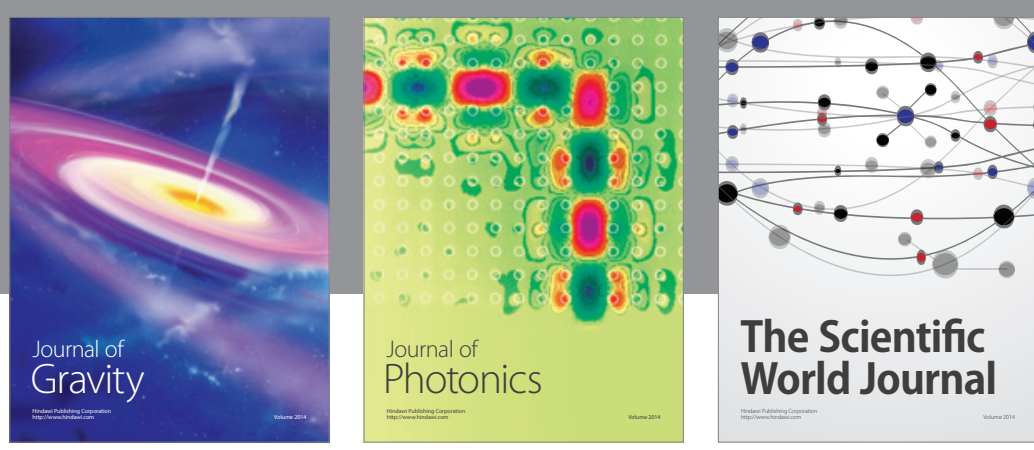

The Scientific World Journal
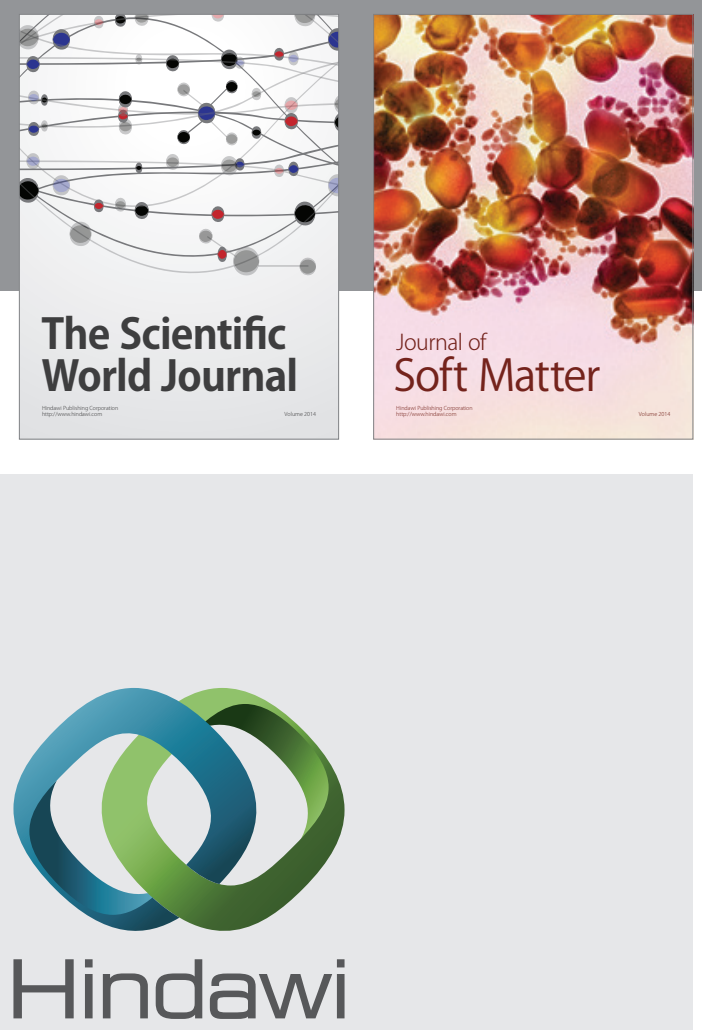

Submit your manuscripts at

https://www.hindawi.com
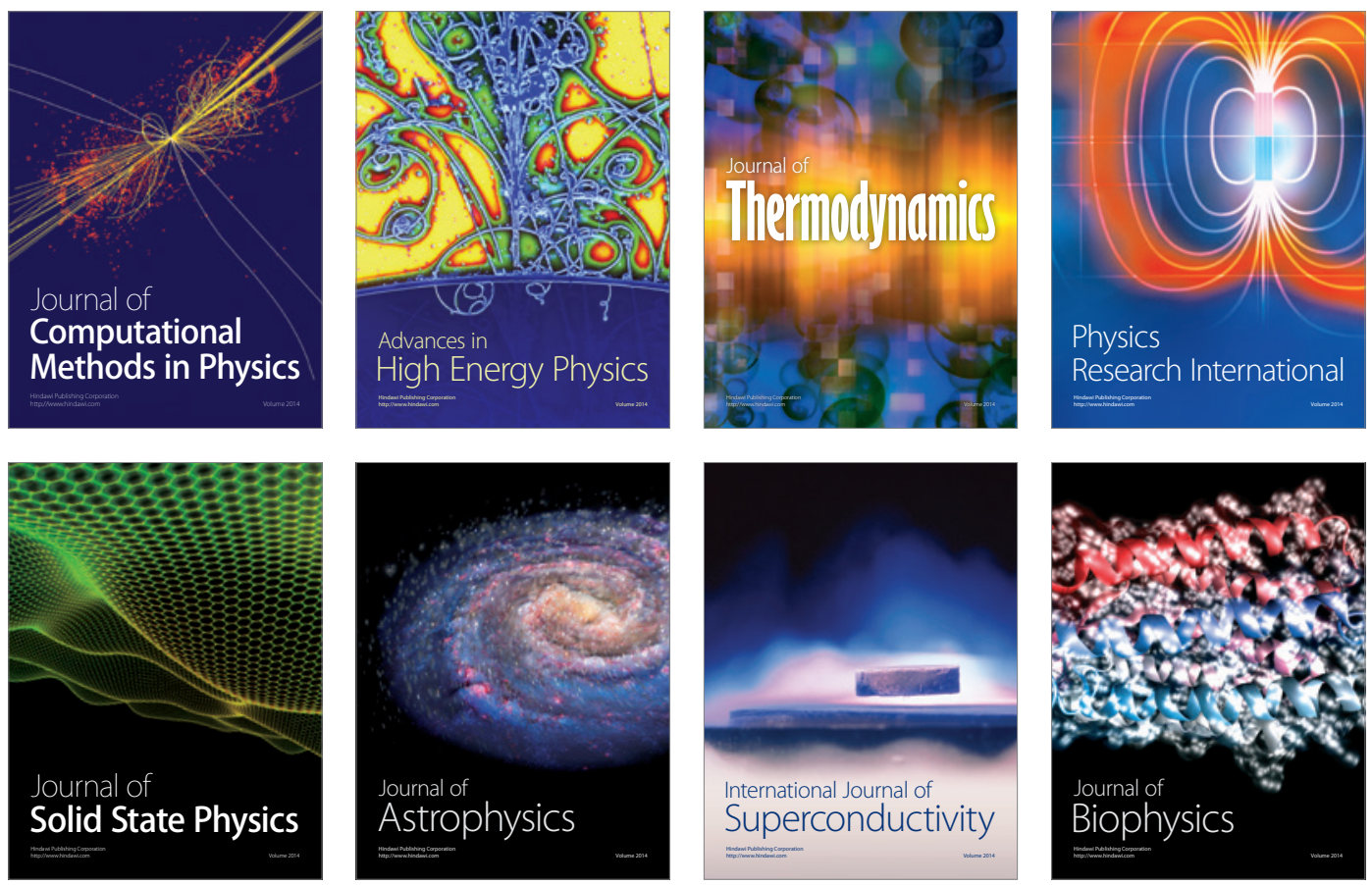
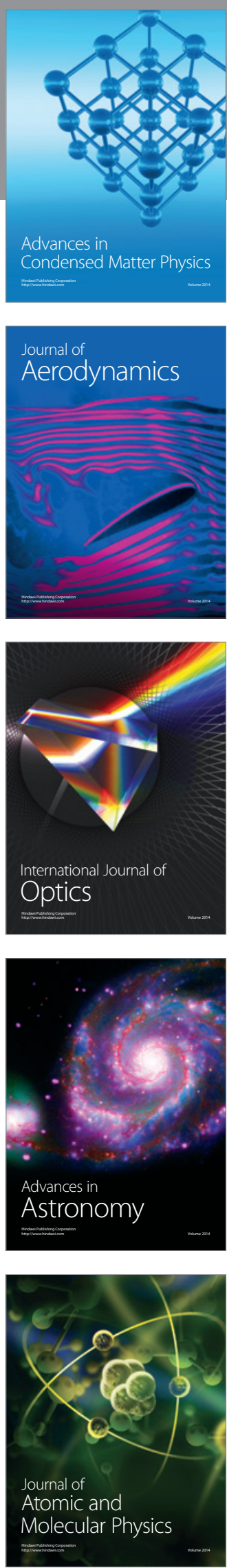